\title{
Multi-Mesh-Scale Approximation of Thin Geophysical Mass Flows on Complex Topographies
}

\author{
Yih-Chin Tai ${ }^{1, *}$, Jeaniffer Vides ${ }^{2}$, Boniface Nkonga $^{3}$ and \\ Chih-Yu Kuo ${ }^{4}$ \\ ${ }^{1}$ Department of Hydraulic and Ocean Engineering, National Cheng Kung \\ University, Tainan, Taiwan. \\ ${ }^{2}$ LEMMA RED Services, Sophia Antipolis, Biot, France $\mathcal{E}$ Maison de la Simulation, \\ Gif-sur-Yvette, France. \\ ${ }^{3}$ Université Côte d'Azur (UCA), LJAD Nice E Inria Sophia Antipolis-Méditerranée, \\ France \\ ${ }^{4}$ Research Center for Applied Sciences, Academia Sinica, Taipei, Taiwan.
}

Received 23 October 2019; Accepted (in revised version) 16 May 2020

\begin{abstract}
This paper is devoted to a multi-mesh-scale approach for describing the dynamic behaviors of thin geophysical mass flows on complex topographies. Because the topographic surfaces are generally non-trivially curved, we introduce an appropriate local coordinate system for describing the flow behaviors in an efficient way. The complex surfaces are supposed to be composed of a finite number of triangle elements. Due to the unequal orientation of the triangular elements, the distinct flux directions add to the complexity of solving the Riemann problems at the boundaries of the triangular elements. Hence, a vertex-centered cell system is introduced for computing the evolution of the physical quantities, where the cell boundaries lie within the triangles and the conventional Riemann solvers can be applied. Consequently, there are two mesh scales: the element scale for the local topographic mapping and the vertex-centered cell scale for the evolution of the physical quantities. The final scheme is completed by employing the HLL-approach for computing the numerical flux at the interfaces. Three numerical examples and one application to a large-scale landslide are conducted to examine the performance of the proposed approach as well as to illustrate its capability in describing the shallow flows on complex topographies.
\end{abstract}

AMS subject classifications: 35L65, 76M12, 58J90, 86-08

Key words: Multi-mesh-scale approach, complex topography, shallow flows, unstructured mesh, vertex-centered formulation. ${ }^{*}$ Corresponding author. Email addresses: yctai@mail.ncku.edu.tw (Y.-C. Tai), jeaniffer.vides@lemma
-ing.com (J. Vides), Boniface.Nkonga@unice.fr (B. Nkonga), cykuo06@gate.sinica.edu.tw (C.-Y. Kuo) 


\section{Introduction}

Most geophysical hazardous flows take place over complex topographies. The occurrences of avalanches, landslides or debris flows over non-trivial topographies in mountainous areas are some clear examples. Not surprisingly, there is a strong link between the particular flow path and the geometry of the underlying basal topography. In addition, these flows are generally "thin" (in depth) compared with the large extension in their tangential direction, so that the term "shallow flow" is employed here to characterize thin flows on curved surfaces. For this type of flows, it is customary to make use of a depth-integration process together with the shallowness assumption to asymptotically derive reduced models for the evolution of the depth-averaged velocity and the thickness of the flow. Conventionally, these models are given in Cartesian coordinates, where the topography is added on a horizontal/inclined plane, or in the so-called "locally inclined Cartesian-type" coordinates [10]. With this approach, many successful models with variant rheological relations, with single-phase [1,2], quasi two-phase [3-7], two- or multi-phase (solid, fine solid and fluid) concepts [8-12], have been proposed and have made fruitful achievements. In addition to the ease of imposing constitutive relations, one of the advantages of applying this geometrically simple coordinate system is the less complicated process of its numerical implementation, so that numerical applications are mostly built based on models with this approach. For example, an advanced GIS-supported open-source computational tool, r.avaflow [13-15], has been developed in recent years for describing two-phase (solid and fluid) or multi-phase (solid, fine solid and fluid) flows over complex topographies with respect to the digital elevation map (DEM) by applying the models proposed by [10] and [12], respectively. However, as the depth-averaged models generally employ the shallowness assumption, in which the computed velocities are parallel to the corresponding coordinate axes instead of the exact basal surface, a high variation in topography leads to significant deviations in representing the depth-averaged velocity. To correct this shortcoming, Savage and Hutter [16] first applied a 2D curvilinear coordinate system aligned with the curved bed for modeling granular flows. This 2D curvilinear coordinate system is extended to 3D by introducing a simple reference surface curved in the down-slope direction, and the complex topography is described by superposing a shallow basal topography on it [17-20]. Although the concept of a simple curved reference plane has also been widely adopted, it suffers from the limitation of the predefined unique down-slope direction of the simple curved reference surface, especially in its application to a snaking canyon terrain. Pudasaini and Hutter [21] improved it by imposing a curved and twisted coordinate system, in which the thalweg coincides with the projection of the master curve on a reference surface. The models presented by curved and twisted coordinate system are advanced and rather successful for the flows over generally curved channels [21-23]. However, determining the necessary master curve(s) and the associated reference surface(s) for complex canyon terrains is not always straightforward.

Bouchut and Westdickenberg (BW) [24] proposed the shallow water equations in a 
general coordinate system, for which there is no need to pre-assign the unique downslope direction nor to determine the master curves. It provides a distinct way of thinking in modeling shallow flows over non-trivial topographic surfaces, e.g., [25-27]. Following BW's approach [24] and integrating its concept with the unified coordinate (UC) method [28,29], a non-conventional approach is proposed for modeling geophysical mass flows in $[30,31]$. The non-conventional approach simplifies the complicate computation caused by the Christoffel symbols in the model and allows the topography variation caused by erosion or deposition [30-34]. With this non-conventional description, an application to a real event of large scale is illustrated in [35]. Recently, the single-phase model employed in [35] has been extended to a two-phase approach [36], in which the phase separation and sediment-concentrated snout, as also illustrated in [11], can be well reproduced. Taking into account the non-hydrostatic (excess) pore-water pressure for the fluid constituent [37,38] and hypoplastic intergranular stress, Heß et al. [39] amended two additional fields and proposed the model equations in the general coordinate system.

The above mentioned models are mainly hyperbolic and finite volume methods are often used for their numerical approximation. In general, the approximation strategies are structured as follows:

- Construction of a global coordinate system, relying on the assumption that the surface description can be defined analytically;

- Representation of the conservative laws, relative to the global coordinate system;

- Reduction of the model equations with the shallowness assumption and scaling analysis;

- Representation of the surface with a finite number of elements;

- Approximation of the reduced model using the discrete surface.

In the context of engineering applications, it is presumptuous to expect that an analytical formulation of the ground surface is attainable. However, from data provided by geographic information systems (GIS), one can casually extract a discrete description of the surfaces that drive thin flows. As a result, it is more practical to use this discrete description as the starting point of the resolution strategy, and this is the kind of approach that will be adopted in this work. In contrast to the quadrilateral meshes used in the numerical implementation [25,33-36], the triangular meshes are employed to fit the non-trivial topography in the present study. That is, the complex surfaces are supposed to be composed of a finite number of triangle elements, and the coordinate system is locally introduced at each triangle element. The main benefit of utilizing the approach of triangular elements is its flexibility of delineating the complex geometry of a rugged topography. The other advantage is the unique orientation (e.g. the normal vector) of the triangular element when comparing the quadrilateral meshes on a non-trivial curved surface. Third, the surface area of a triangular element can be easily computed, while the 
quadrilateral mesh is generally not of the shape of a parallelogram. However, the unequal orientation of the triangular elements may enhance the complexity by solving the Riemann problem at the boundaries between the elements. Hence a vertex-centered cell system is employed. That is, the evolution of the physical quantities are computed with respect to the vertex-centered cell, where the cell boundaries lie within the triangles and the conventional Riemann solvers can be well utilized. So, the proposed approach locally consists of two mesh scales: the element scale (the triangular element) and the cell scale (the vertex-centered cell), cf. Fig. 1. The discrete mapping and the reduced model are both defined at the element scale, and the averaged values that evolve in time are defined at the cell scale $[40,41]$. With this multi-mesh-scale approximation there is no need to define a non-singular global mapping (1-to-1 mapping between the vertical-horizontal-oriented Cartesian coordinates and the terrain-fitted coordinates), e.g. a spiral channel. Recently, a similar concept has been proposed in [42]. Because it is a curvature-free approach, the curvature-induced centrifugal force amendment to the momentum equation is added posteriorly in the simulation, but the impact of the velocity direction on the curvature in the $3 \mathrm{D}$ space is regrettably not taken into account. The present formulation includes the complete topographical curvature tensor, such that the three-dimensional characteristics of the curvature and the flow velocity direction dependency are retained.

In Section 2, we present the approach, which is a discrete sub-scale formulation and consists of 6 subsections, from the "parameterization of the local triangular element" to the "final scheme for shallow flows". Next, the developed strategy is validated in Section 3 and applied to a real event, back-calculating the plausible flow process, in Section 4. The concluding remarks are given in the last section.

\section{Discrete sub-scale formulation and reduction}

Let us start by considering a surface $\Gamma \in \mathbb{R}^{3}$ that represents the basal topographic surface. Formally, we have

$$
\Gamma=\left\{\overline{\widetilde{x}}=\left(\begin{array}{c}
x \\
b
\end{array}\right) \in \mathbb{R}^{3}, \text { where } \boldsymbol{x}=\left(\begin{array}{l}
x \\
y
\end{array}\right) \in \Omega \subset \mathbb{R}^{2} \text { and } b=b(\boldsymbol{x})\right\},
$$

with $x$ lying on the horizontal plane and $z=b(\boldsymbol{x})$ the equation defining the basal surface. In practice, this description is generally given in discrete form, such as by the Digital Elevation Maps (DEMs). In this regard, we settle for approximated surfaces that are piecewise defined:

$$
\Gamma_{h}=\bigcup_{e} \mathcal{E}_{e} \simeq \Gamma
$$

where $\mathcal{E}_{e} \equiv\left(\overline{\widetilde{\boldsymbol{x}}}_{i}, \overline{\widetilde{\boldsymbol{x}}}_{j}, \overline{\boldsymbol{x}}_{k}\right)$ are non-overlapping flat triangular surfaces defined by the points

$$
\overline{\tilde{\boldsymbol{x}}}_{i}=\left(\begin{array}{c}
\boldsymbol{x}_{i} \\
b\left(\boldsymbol{x}_{i}\right)
\end{array}\right), \quad \overline{\boldsymbol{x}}_{j}=\left(\begin{array}{c}
\boldsymbol{x}_{j} \\
b\left(\boldsymbol{x}_{j}\right)
\end{array}\right) \quad \text { and } \quad \overline{\tilde{\boldsymbol{x}}}_{k}=\left(\begin{array}{c}
\boldsymbol{x}_{k} \\
b\left(\boldsymbol{x}_{k}\right)
\end{array}\right) \text {. }
$$




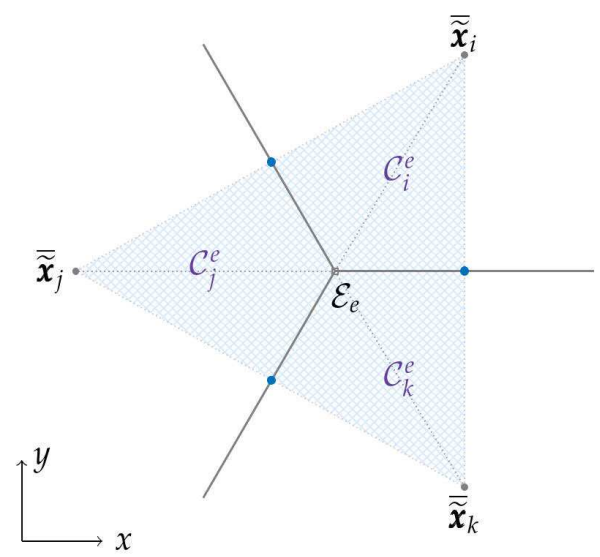

(a) Triangular element $\mathcal{E}_{e}$

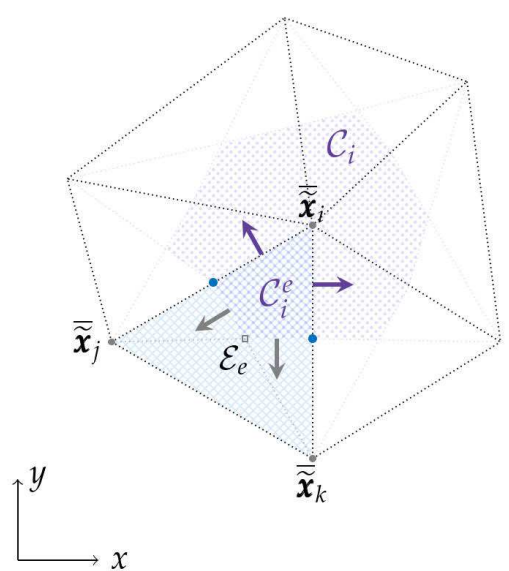

(b) Vertex-centered cell $\mathcal{C}_{i}$

Figure 1: Sketches of a triangular surface element $\mathcal{E}_{e}$ (panel a) and a vertex-centered cell $\mathcal{C}_{i}$ (panel b), where the element $\mathcal{E}_{e}$ consists of three sub-cells, $\mathcal{C}_{i}^{e}, \mathcal{C}_{j}^{e}$ and $\mathcal{C}_{k}^{e}$, and the sub-cell $\mathcal{C}_{i}^{e}$ is associated to both $\mathcal{C}_{i}$ and $\mathcal{E}_{e}$, i.e., $\mathcal{C}_{i}^{e}=\mathcal{C}_{i} \cap \mathcal{E}_{e}$.

This strategy for the representation of the basal surface is similar to the one used in $[24,33,34,43]$, but therein the numerical strategy considers the control volumes to be the triangles (cell-centered approach). Instead, in this work we make use of dual cells in a vertex-centered formulation. For each element $\mathcal{E}_{e}$, three sub-cells can be obtained by connecting the centroid of the element's face with the midpoints of the edges, $\mathcal{E}_{e}=$ $\mathcal{C}_{i}^{e} \cup \mathcal{C}_{j}^{e} \cup \mathcal{C}_{k}^{e}$ as depicted in Fig. 1(a). Thus, by using the element scale, the cell scale is obtained from the union of the sub-cells associated to the mesh vertex and its neighboring elements (see Fig. 1(b)). Each control cell $\mathcal{C}_{i}$ is then defined as

$$
\mathcal{C}_{i}=\bigcup_{e \in \mathcal{E}(i)} \mathcal{C}_{i}^{e}
$$

Note that $\mathcal{C}_{i}$ is the union of planar polygons, but the cell itself is generally non-planar. Examples of non-planar dual cells on $\boldsymbol{\Gamma}_{h} \subset \mathbb{R}^{2}$ (section view) and $\boldsymbol{\Gamma}_{h} \subset \mathbb{R}^{3}$ are shown in Figs. 2 and 3 , respectively. In the latter, one can clearly see that the vertices of $\mathcal{C}_{i}$ (in purple) do not all lie on the same plane.

\subsection{Parameterization of the local triangular element}

On each triangular surface, we introduce a local system of coordinates $\widetilde{\xi} \equiv \widetilde{\boldsymbol{\xi}}^{e}$; in what follows, we use $\widetilde{\xi}$ instead of $\widetilde{\boldsymbol{\xi}}^{e}$ to simplify our writing. In addition, we introduce the following notation:

$$
\widetilde{\boldsymbol{\xi}}=\left(\begin{array}{c}
\xi \\
\zeta
\end{array}\right), \quad \overline{\widetilde{x}}=\left(\begin{array}{c}
x \\
b(x)
\end{array}\right), \quad \widetilde{\boldsymbol{x}}=\left(\begin{array}{c}
x \\
z
\end{array}\right), \quad \widetilde{\boldsymbol{y}}=\left(\begin{array}{c}
x \\
0
\end{array}\right),
$$




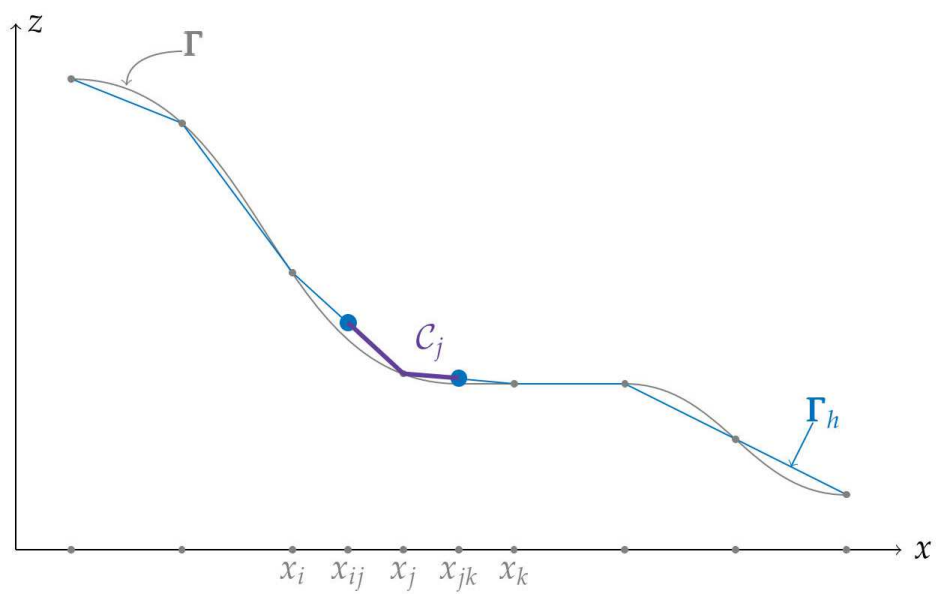

Figure 2: Construction of a non-planar cell $\mathcal{C}_{j}$ for an approximated surface $\Gamma_{h} \in \mathbb{R}^{2}$.

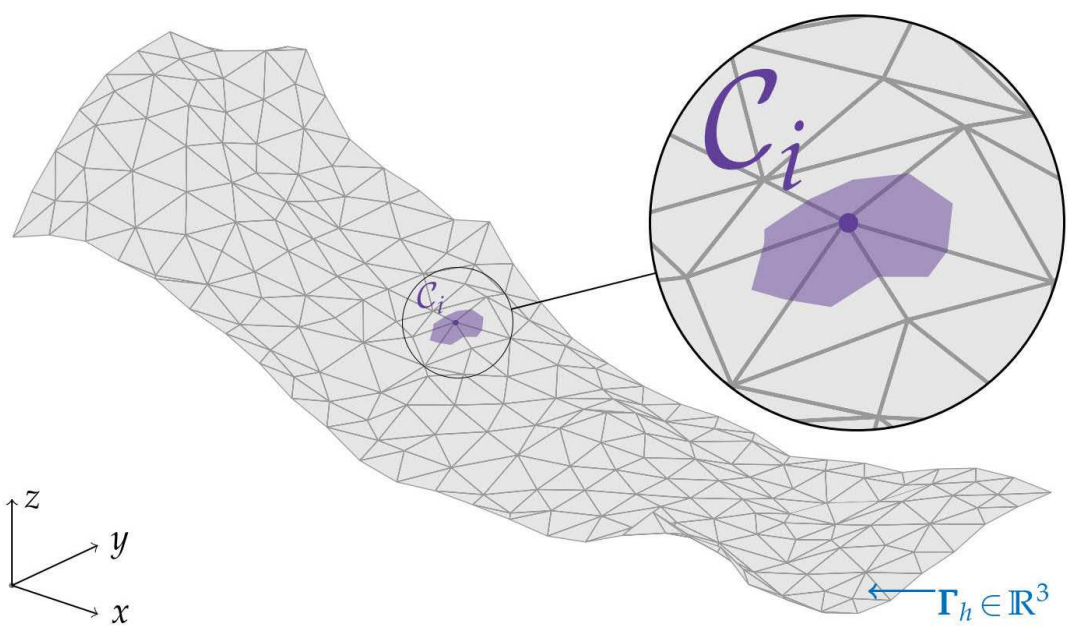

Figure 3: Construction of $\mathcal{C}_{i}$ (in purple) for a surface approximated by a finite number of triangles (in gray). The cell $\mathcal{C}_{i}$ is centered on the vertex $i$ and it is non-planar, i.e., its points do not lie on the same plane. As shown in the zoomed panel, the vertex-centered cell is a dual of the element cells (see also Fig. 1b).

all specified in terms of the coordinate pairs

$$
\boldsymbol{x}=\left(\begin{array}{l}
x \\
y
\end{array}\right) \quad \text { and } \boldsymbol{\xi}=\left(\begin{array}{l}
\xi \\
\eta
\end{array}\right)
$$

In (2.1a), we have purposely written $\overline{\widetilde{x}}=(x, y, b(x, y))^{T}$ once more to point out the difference between it and $\widetilde{\boldsymbol{x}}=(x, y, z)^{T}$, i.e., the latter refers to the Cartesian coordinates in the three-dimensional space and the former to a point on the topographic surface. The basal 


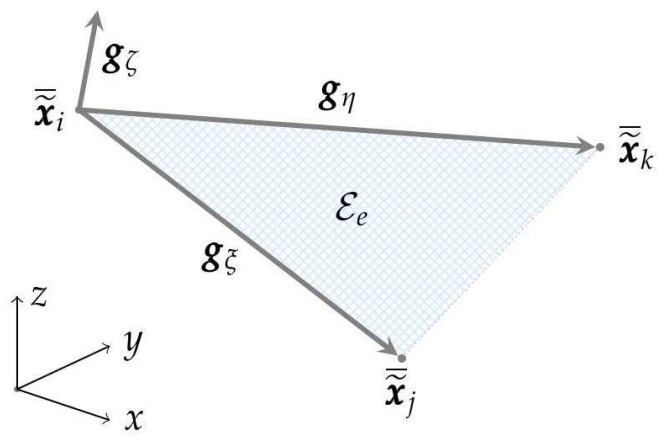

Figure 4: The local basis associated to a triangular element $\mathcal{E}_{\mathcal{e}}$.

surface on an element $\mathcal{E}$ is defined as

$$
b(\boldsymbol{x})=b_{i}+\left(b_{j}-b_{i}\right) \Lambda_{j}(\boldsymbol{x})+\left(b_{k}-b_{i}\right) \Lambda_{k}(\boldsymbol{x}),
$$

being $\Lambda_{m}(\boldsymbol{x})$ (linear) barycentric coordinate functions on the Cartesian coordinates. For instance, $\Lambda_{i}(\boldsymbol{x})=1-\Lambda_{j}(\boldsymbol{x})-\Lambda_{k}(\boldsymbol{x})$ with

$$
\Lambda_{j}(\boldsymbol{x})=\frac{\left[\left(\widetilde{\boldsymbol{y}}(\boldsymbol{x})-\widetilde{\boldsymbol{y}}_{i}\right) \times\left(\widetilde{\boldsymbol{y}}_{k}-\widetilde{\boldsymbol{y}}_{i}\right)\right] \cdot \widehat{\boldsymbol{e}}_{z}}{\left[\left(\widetilde{\boldsymbol{y}}_{j}-\widetilde{\boldsymbol{y}}_{i}\right) \times\left(\widetilde{\boldsymbol{y}}_{k}-\widetilde{\boldsymbol{y}}_{i}\right)\right] \cdot \widehat{\boldsymbol{e}}_{z}}, \quad \Lambda_{k}(\boldsymbol{x})=\frac{\left[\left(\widetilde{\boldsymbol{y}}(\boldsymbol{x})-\widetilde{\boldsymbol{y}}_{i}\right) \times\left(\widetilde{\boldsymbol{y}}_{j}-\widetilde{\boldsymbol{y}}_{i}\right)\right] \cdot \widehat{\boldsymbol{e}}_{z}}{\left[\left(\widetilde{\boldsymbol{y}}_{k}-\widetilde{\boldsymbol{y}}_{i}\right) \times\left(\widetilde{\boldsymbol{y}}_{j}-\widetilde{\boldsymbol{y}}_{i}\right)\right] \cdot \widehat{\boldsymbol{e}}_{z}},
$$

where $\left(\widehat{\boldsymbol{e}}_{x}, \widehat{\boldsymbol{e}}_{y}, \widehat{\boldsymbol{e}}_{z}\right)$ is the standard Cartesian basis (in $\mathbb{R}^{3}$ ) and $\widetilde{\boldsymbol{y}}$ the Cartesian coordinates projected on the plane $z=0$, as defined in (2.1).

As illustrated in Fig. 4, the local basis associated to an element is defined by the vectors $\boldsymbol{g}_{\tilde{\zeta}}, \boldsymbol{g}_{\eta}$ and $\boldsymbol{g}_{\zeta}$, where

$$
\boldsymbol{g}_{\xi}=\overline{\widetilde{\boldsymbol{x}}}_{j}-\overline{\overline{\boldsymbol{x}}}_{i}, \quad \boldsymbol{g}_{\eta}=\overline{\widetilde{\boldsymbol{x}}}_{k}-\overline{\widetilde{\boldsymbol{x}}}_{i} \quad \text { and } \quad \boldsymbol{g}_{\zeta}=\frac{\boldsymbol{g}_{\xi} \times \boldsymbol{g}_{\eta}}{\left\|\boldsymbol{g}_{\xi} \times \boldsymbol{g}_{\eta}\right\|},
$$

and the local transformation on an element is

$$
\widetilde{\boldsymbol{x}}(\widetilde{\boldsymbol{\xi}})=\xi \boldsymbol{g}_{\xi}+\eta \boldsymbol{g}_{\eta}+\zeta \boldsymbol{g}_{\zeta}
$$

The term $\zeta$ can be regarded as the distance above the basal surface; on the basal surface itself, $\zeta=\zeta_{e}=0$. The vectors $\boldsymbol{g}_{\xi}, \boldsymbol{g}_{\eta}$ and $\boldsymbol{g}_{\zeta}$ are the covariant ones associated to the transformation. As these local vectors are constant, we have

$$
\boldsymbol{g}_{\xi}=\partial_{\tilde{\zeta}} \widetilde{\boldsymbol{x}}, \quad \boldsymbol{g}_{\eta}=\partial_{\eta} \widetilde{\boldsymbol{x}} \text { and } \boldsymbol{g}_{\zeta}=\partial_{\zeta} \widetilde{\boldsymbol{x}},
$$

and the Jacobian of the transformation

$$
\mathrm{J}=\operatorname{det}\left(\frac{\partial}{\partial \widetilde{\boldsymbol{\xi}}} \widetilde{\boldsymbol{x}}\right)=\left(\boldsymbol{g}_{\xi} \times \boldsymbol{g}_{\eta}\right) \cdot \boldsymbol{g}_{\zeta}
$$


This transformation is compatible with the ones proposed in $[24,25,32,34]$, but with the simplification that the current transformation is locally linear, i.e., within the triangular element $\mathcal{E}_{e}$. As a consequence, the Jacobian and transformation matrices are constant. Indeed, simple calculations show that

$$
\frac{\partial}{\partial \widetilde{\boldsymbol{\xi}}} \widetilde{\boldsymbol{x}}=\left(\boldsymbol{g}_{\tilde{\zeta}}, \boldsymbol{g}_{\eta}, \boldsymbol{g}_{\zeta}\right)=\left(\begin{array}{cc|c}
\partial_{\tilde{\xi}} x & \partial_{\eta} x & -c \partial_{x} b \\
\partial_{\xi} y & \partial_{\eta} y & -c \partial_{y} b \\
\hline \partial_{\xi} b & \partial_{\eta} b & c
\end{array}\right)=\left(\begin{array}{c|c}
\partial_{\xi} x & -s \\
\hline \frac{1}{c} \boldsymbol{s}^{\mathrm{T}} \partial_{\xi} \boldsymbol{x} & c
\end{array}\right),
$$

with

$$
c=\frac{1}{\sqrt{1+\left\|\partial_{x} b\right\|^{2}}}, \quad s=c \partial_{x} b \quad \text { and } \quad \frac{1}{c} s^{\mathrm{T}} \partial_{\xi} x=\left(\partial_{\xi} b, \partial_{\eta} b\right)
$$

see $[24,32,34]$. The rows of the inverse matrix $\frac{\partial}{\partial \widetilde{x}} \widetilde{\boldsymbol{\xi}} \equiv\left(\frac{\partial}{\partial \widetilde{\xi}} \widetilde{\boldsymbol{x}}\right)^{-1}$ are the contravariant vectors $\boldsymbol{g}^{\xi}, \boldsymbol{g}^{\eta}$ and $\boldsymbol{g}^{\zeta}$, specifically

$$
\left(\frac{\partial}{\partial \widetilde{\boldsymbol{x}}} \widetilde{\xi}\right)^{\mathrm{T}}=\left(\boldsymbol{g}^{\xi}, \boldsymbol{g}^{\eta}, \boldsymbol{g}^{\zeta}\right)=\left(\begin{array}{c|c}
(\underline{\mathbf{I}}-\mathbf{s} \otimes \boldsymbol{s})\left(\partial_{\boldsymbol{x}} \boldsymbol{\xi}\right)^{\mathrm{T}} & -\boldsymbol{s} \\
\hline c \boldsymbol{s}^{\mathrm{T}}\left(\partial_{\boldsymbol{x}} \boldsymbol{\xi}\right)^{\mathrm{T}} & c
\end{array}\right),
$$

where they follow the relations $\boldsymbol{g}_{n} \cdot \boldsymbol{g}^{p}=\delta_{n}^{p}$ with $\delta_{n}^{p}$ being the Kronecker delta.

We now have the choice to use hereafter either the matrices and sub-matrices of the transformation (as in [24]) or covariant and contravariant vectors (as was partially the case in [34]). For simplicity of the formulation, we subsequently employ the covariant and contravariant vectors and assume that the basal surface does not change with time. Then, for any vector $\mathbf{V}$, the divergence $\nabla_{\widetilde{x}} \cdot \mathbf{V}$ in the Cartesian coordinates takes the following form with curvilinear coordinates:

$$
\mathrm{J} \nabla_{\widetilde{\boldsymbol{x}}} \cdot \mathbf{V}=\partial_{\tilde{\zeta}}\left(\mathrm{J} \mathbf{V} \cdot \boldsymbol{g}^{\tau}\right)+\partial_{\eta}\left(\mathrm{JV} \cdot \boldsymbol{g}^{\eta}\right)+\partial_{\zeta}\left(\mathrm{JV} \cdot \boldsymbol{g}^{\zeta}\right)=\nabla_{\widetilde{\boldsymbol{\xi}}} \cdot\left(\mathrm{J}\left(\partial_{\widetilde{x}} \widetilde{\boldsymbol{\xi}}\right) \mathbf{v}\right)
$$

Moreover, for any symmetric tensor field $\underline{\mathbf{T}}$ and a linear transformation (see, for instance, [24, Lemma 3.1, page 28]), we have

$$
\mathrm{J} \nabla_{\widetilde{\boldsymbol{x}}} \cdot \underline{\mathbf{T}}=\partial_{\tilde{\xi}}\left(\mathrm{J} \underline{\mathbf{T}} \boldsymbol{g}^{\xi}\right)+\partial_{\eta}\left(\mathrm{J} \underline{\mathbf{T}} g^{\eta}\right)+\partial_{\zeta}\left(\mathrm{J} \underline{\mathbf{T}} \boldsymbol{g}^{\zeta}\right)=\nabla_{\widetilde{\xi}} \cdot(\mathrm{J} \underline{\mathbf{T}} \underline{\mathbf{K}}),
$$

with $\underline{\mathbf{K}}=\left(\partial_{\widetilde{\boldsymbol{x}}} \widetilde{\boldsymbol{\xi}}\right)^{\mathrm{T}}$. Thus, the divergence theorem in curvilinear coordinates reads

$$
\int_{\Omega} \nabla_{\widetilde{\boldsymbol{\xi}}} \cdot\left(\mathrm{J}\left(\partial_{\widetilde{\boldsymbol{x}}} \widetilde{\boldsymbol{\xi}}\right) \mathbf{V}\right) d \widetilde{\boldsymbol{\xi}}=\int_{\partial \Omega}\left(\mathrm{J}\left(\partial_{\tilde{\boldsymbol{x}}} \widetilde{\boldsymbol{\xi}}\right) \mathbf{v}\right) \cdot \mathrm{N} d \widetilde{S}_{\widetilde{\boldsymbol{\xi}}}=\int_{\partial \Omega} \mathrm{J} \mathbf{V} \cdot \widetilde{\boldsymbol{n}} d \widetilde{S}_{\widetilde{\boldsymbol{\xi}}}
$$

for the vector $\mathbf{V}$, and

$$
\int_{\Omega} \nabla_{\widetilde{\boldsymbol{\xi}}} \cdot(\mathbf{J} \underline{\mathbf{T}} \underline{\mathbf{K}}) d \widetilde{\boldsymbol{\xi}}=\int_{\partial \Omega} \mathbf{J} \underline{\mathbf{T}} \widetilde{\boldsymbol{n}} d \widetilde{S}_{\widetilde{\boldsymbol{\xi}}},
$$

for the tensor $\underline{\mathbf{T}}$, where the relation $\widetilde{\boldsymbol{n}} d \widetilde{S}_{\widetilde{\boldsymbol{\zeta}}} \equiv \underline{\mathbf{K}} \mathrm{N} d \widetilde{S}_{\widetilde{\boldsymbol{\zeta}}}$ is detailed in Appendix A.1. 


\subsection{Local numerical model of the vertex-centered scale}

In order to use the most suitable reduced model for each element, it is desirable to also formulate the velocity field in the covariant and contravariant vectors, i.e.,

$$
\mathbf{U}=u_{x} \widehat{\boldsymbol{e}}_{x}+u_{y} \widehat{\boldsymbol{e}}_{y}+u_{z} \widehat{\boldsymbol{e}}_{z}=u_{\zeta} \boldsymbol{g}^{\xi}+u_{\eta} \boldsymbol{g}^{\eta}+u_{\zeta} \boldsymbol{g}^{\zeta}=u^{\zeta} \boldsymbol{g}_{\xi}+u^{\eta} \boldsymbol{g}_{\eta}+u^{\zeta} \boldsymbol{g}_{\zeta}
$$

for which one can notice that $u^{\xi}=\mathbf{U} \cdot \boldsymbol{g}^{\xi}, u^{\eta}=\mathbf{U} \cdot \boldsymbol{g}^{\eta}$ and $u^{\zeta}=\mathbf{U} \cdot \boldsymbol{g}^{\zeta}$. In the local frame, the mass and momentum conservative equations for an incompressible fluid read

$$
\left\{\begin{array}{l}
\frac{\partial}{\partial \xi}\left(\mathrm{J} u^{\xi}\right)+\frac{\partial}{\partial \eta}\left(\mathrm{J} u^{\eta}\right)+\frac{\partial}{\partial \zeta}\left(\mathrm{J} u^{\zeta}\right)=0 \\
\frac{\partial}{\partial t}(\mathrm{~J} \mathbf{U})+\frac{\partial}{\partial \xi}\left[\mathrm{J}\left(\mathbf{U} u^{\xi}+\underline{\mathbf{P}} \boldsymbol{g}^{\xi}\right)\right]+\frac{\partial}{\partial \eta}\left[\mathrm{J}\left(\mathbf{U} u^{\eta}+\underline{\mathbf{P}} \boldsymbol{g}^{\eta}\right)\right]+\frac{\partial}{\partial \zeta}\left[\mathrm{J}\left(\mathbf{U} u^{\zeta}+\underline{\mathbf{P}} \boldsymbol{g}^{\zeta}\right)\right]=0
\end{array}\right.
$$

where $\underline{\mathbf{P}}=\frac{1}{\rho}\left[\left(p+\rho g\left(z-z_{0}\right)\right) \underline{\mathbf{I}}-\underline{\boldsymbol{\tau}}\right]$ is a symmetric tensor representing the generalized stress tensor containing the gravity and $\rho$ is the fluid density. These equations are in conservative form and are therefore suitable for finite volume approximations.

\subsubsection{Integration over the sub-cell $\mathcal{C}_{i}^{e}$}

The considered flow is a free surface flow and we assume that on the sub-cell $\mathcal{C}_{i}^{e}$ (cf. Fig. 1), it has a height $h^{e}(t, \tilde{\xi})$ in the normal direction $\boldsymbol{g}^{\zeta}$. The integration of system (2.7) over the local volume $\mathcal{V}_{i}^{e}(t, \widetilde{\boldsymbol{\xi}})=\bigcup_{\boldsymbol{\xi}} \mathcal{C}_{i}^{e}(\boldsymbol{\xi}) \times\left[0, h^{e}(t, \boldsymbol{\xi})\right]$ yields

$$
\begin{aligned}
& \int_{\partial \mathcal{V}_{i}^{e}} \mathrm{U} \cdot \widetilde{\boldsymbol{n}} d \sigma=0, \\
& \frac{\partial}{\partial t}\left(\int_{\mathcal{V}_{i}^{e}} \mathrm{~J} \mathbf{U} d \boldsymbol{\xi} d \zeta\right)-\int_{\mathcal{C}_{i}^{e}} \mathrm{JU} \partial_{t} h^{e} d \boldsymbol{\xi}+\int_{\partial \mathcal{V}_{i}^{e}} \mathrm{~J}(\mathbf{U U} \cdot \widetilde{\boldsymbol{n}}+\underline{\mathbf{P}} \widetilde{\boldsymbol{n}}) d \sigma=0,
\end{aligned}
$$

with $\partial \mathcal{V}_{i}^{e}$ the boundary of the volume $\mathcal{V}_{i}^{e}$. The boundary $\partial \mathcal{V}_{i}^{e}$ can be decomposed into four parts associated (i) to the basal surface, (ii) to the free surface, (iii) to interactions with the other two neighboring cells (within the triangular element $\mathcal{E}_{e}$ ), and (iv) to the boundary $\partial e$ of the element $e$ (within the vertex-centered cell $\mathcal{C}_{i}$ ). That is,

$$
\begin{aligned}
& \partial \mathcal{V}_{i}^{e}=\underbrace{\left(\bigcup_{\boldsymbol{\xi}} \mathcal{C}_{i}^{e}(\boldsymbol{\xi}) \times\{0\}\right)}_{(\mathrm{i})} \bigcup \underbrace{\left(\bigcup_{\boldsymbol{\xi}} \mathcal{C}_{i}^{e}(\boldsymbol{\xi}) \times\left\{h^{e}(t, \boldsymbol{\xi})\right\}\right)}_{(\mathrm{ii})} \\
& \bigcup_{j \in \vartheta(i, e)} \underbrace{\left(\bigcup_{\xi}^{(i)} \partial \mathcal{C}_{i}^{e} \cap \partial \mathcal{C}_{j}^{e} \times\left[0, h^{e}(t, \boldsymbol{\xi})\right]\right)^{(i i)}}_{(\text {iii) }} \bigcup \underbrace{\left(\bigcup_{\xi} \partial \mathcal{C}_{i}^{e} \cap \partial e \times\left[0, h^{e}(t, \boldsymbol{\xi})\right]\right)}_{\text {(iv) }},
\end{aligned}
$$


where $\vartheta(i, e)$ is the sub-set of cells neighbors to the cell $\mathcal{C}_{i}$ and associated to the element $e$. An integral over this boundary is therefore the sum of integrals over each of the abovementioned parts. Let us now turn our attention to these surface integrals.

(i) Integrals associated to the basal surface: $\zeta=0$

The normal vector at the basal surface is simply defined as $\widetilde{\boldsymbol{n}} d \sigma=-\boldsymbol{g}^{\zeta} d \boldsymbol{\xi}$. Then, the integrals over $\mathcal{C}_{i}^{e}(\boldsymbol{\xi}) \times\{0\}$ become

$$
\begin{aligned}
& \int_{\mathcal{C}_{i}^{e}(\boldsymbol{\xi}) \times\{0\}} \mathrm{JU} \cdot \widetilde{\tilde{n}} d \sigma=-\int_{\mathcal{C}_{i}^{e}}\left(\mathrm{~J} u^{\zeta}\right)_{\zeta=0} d \boldsymbol{\xi}, \\
& \int_{\mathcal{C}_{i}^{e}(\boldsymbol{\xi}) \times\{0\}} \mathrm{J}(\mathbf{U U} \cdot \widetilde{\boldsymbol{n}}+\underline{\mathbf{P}} \widetilde{\boldsymbol{n}}) d \sigma=-\int_{\mathcal{C}_{i}^{e}} \mathrm{~J}\left(\mathbf{U} u^{\zeta}+\underline{\mathbf{P}} \boldsymbol{g}^{\zeta}\right)_{\zeta=0} d \boldsymbol{\xi} .
\end{aligned}
$$

(ii) Integrals associated to the free surface: $\zeta=h^{e}(t, \boldsymbol{\xi})$

The normal vector at the free surface is such that $\widetilde{\boldsymbol{n}} d \sigma=\mathrm{N} d \boldsymbol{\xi}$, with $\mathrm{N}=\boldsymbol{g}^{\zeta}-\partial_{\xi} h^{e} \boldsymbol{g}^{\xi}-\partial_{\eta} h^{e} \boldsymbol{g}^{\eta}$ (we refer the reader to Appendix A.1 for more details regarding N). The kinematic boundary condition at the free surface can be written as

$$
\partial_{t} h^{e}=\mathrm{N} \cdot \mathrm{U}
$$

Then, we arrive at

$$
\int_{\mathcal{C}_{i}^{e}(\boldsymbol{\xi}) \times\left\{h^{e}\right\}} \mathrm{JU} \cdot \widetilde{\boldsymbol{n}} d \sigma=\int_{\mathcal{C}_{i}^{e}} \mathrm{~J} \partial_{t} h^{e} d \boldsymbol{\xi}=\frac{\partial}{\partial t}\left(\int_{\mathcal{C}_{i}^{\mathrm{J}}} h^{e} d \boldsymbol{\xi}\right),
$$

and

$$
\int_{\mathcal{C}_{i}^{e}(\tilde{\xi}) \times\left\{h^{e}\right\}} \mathrm{J}(\mathbf{U U} \cdot \widetilde{\boldsymbol{n}}+\underline{\mathbf{P}} \widetilde{\boldsymbol{n}}) d \sigma=\int_{\mathcal{C}_{i}^{e}} \mathrm{JU} \partial_{t} h^{e} d \boldsymbol{\xi}+\int_{\mathcal{C}_{i}^{e}} \underline{\mathbf{P}}_{h} \mathrm{~N} d \boldsymbol{\xi} .
$$

Note that the contribution of the integral following the equal sign in Eq. (2.13) will cancel out the contribution associated to the second term of Eq. (2.9).

\section{(iii) Integrals associated to interactions with the neighbor cells}

Let us recall that $\vartheta(i, e)$ is the sub-set of cells neighbors to the cell $i$ and associated to the element $e$. Then, for any $j \in \vartheta(i, e)$, we compute the following fluxes:

$$
\begin{aligned}
\boldsymbol{\phi}_{i j}^{h, e} & =\int_{\partial \mathcal{C}_{i}^{e} \cap \partial \mathcal{C}_{j}^{e}} \int_{0}^{h^{e}} \mathrm{JU} \cdot \widetilde{\boldsymbol{n}} d \zeta \widetilde{d \ell}, \\
\boldsymbol{\phi}_{i j}^{h \mathbf{U}, e} & =\int_{\partial \mathcal{C}_{i}^{e} \cap \partial \mathcal{C}_{j}^{e}} \int_{0}^{h^{e}} \mathrm{~J}(\mathbf{U} \otimes \mathbf{U}+\underline{\mathbf{P}}) \widetilde{\boldsymbol{n}} d \zeta \widetilde{d \ell},
\end{aligned}
$$

where $\widetilde{n}$ is the outward normal vector (the grey arrows in Fig. 1(b)) and will be formulated in the covariant basis later (refer to Appendix A.1). 


\section{(iv) Integrals associated to the boundary $\partial e$ of the element $e$}

They are the internal fluxes within the same vertex-centered cell (indicated by the purple arrows in Fig. 1(b)) and will vanish in the cell-scale approximation. Hence, there is no need to formulate them.

\subsubsection{The discrete formulation: equations for the averaged quantities in the cell $\mathcal{C}_{i}$}

After having obtained proper expressions for the surface integrals, we are now able to continue deriving the discrete model. On this account, let us first introduce the sub-scale variables $\bar{h}_{i}^{e}$ and $\overline{\mathbf{U}}_{i}^{e}(t)$, defined by

$$
\bar{h}_{i}^{e}=\frac{1}{a_{i}^{e}} \int_{\mathcal{C}_{i}^{e}} \mathrm{~J} h^{e} d \boldsymbol{\xi} \quad \text { and } \quad \bar{h}_{i}^{e} \overline{\mathbf{U}}_{i}^{e}(t)=\frac{1}{a_{i}^{e}} \int_{\mathcal{V}_{i}^{e}} \mathrm{~J} \mathbf{U} d \widetilde{\boldsymbol{\xi}}, \quad \text { with } \quad a_{i}^{e}=\int_{\mathcal{C}_{i}^{e}} \mathrm{~J} d \boldsymbol{\xi} .
$$

Then, according to the previously established integrals (2.10)-(2.15), the evolution equations of $\bar{h}_{i}^{e}$ and $\bar{h}_{i}^{e} \overline{\mathbf{U}}_{i}^{e}$, contained in Eqs. (2.8) and (2.9), compose the element-scale approximation and can be reformulated as

$$
\begin{aligned}
\partial_{t}\left(a_{i}^{e} \bar{h}_{i}^{e}\right)+\sum_{j \in \vartheta(i, e)} \boldsymbol{\phi}_{i j}^{h, e}= & \int_{\mathcal{C}_{i}^{e}}\left(\mathrm{~J} u^{\zeta}\right)_{\zeta=0} d \boldsymbol{\xi}-\int_{\partial \mathcal{C}_{i}^{e} \cap \partial e} \int_{0}^{h^{e}} \mathrm{JU} \cdot \widetilde{\boldsymbol{n}} d \zeta d \widetilde{\ell} \\
\partial_{t}\left(a_{i}^{e} \bar{h}_{i}^{e} \overline{\mathbf{U}}_{i}^{e}\right)+\sum_{j \in \vartheta(i, e)} \boldsymbol{\phi}_{i j}^{h \mathbf{U}, e}= & \int_{\mathcal{C}_{i}^{e}} \mathrm{~J}\left(\mathbf{U} u^{\zeta}+\underline{\mathbf{P}} \boldsymbol{g}^{\zeta}\right)_{\zeta=0} d \boldsymbol{\xi}-\int_{\mathcal{C}_{i}^{e}} \mathrm{~J}(\underline{\mathbf{P}} \mathrm{N})_{\zeta=h} d \boldsymbol{\xi} \\
& -\int_{\partial \mathcal{C}_{i}^{e} \cap \partial e} \int_{0}^{h^{e}} \mathrm{~J}(\mathbf{U} \otimes \mathbf{U}+\underline{\mathbf{P}}) \widetilde{\boldsymbol{n}} d \zeta d \widetilde{\ell}
\end{aligned}
$$

Now we introduce the numerical scheme for quantities at the cell scale. For this, we wish to recall that each cell $\mathcal{C}_{i}$ has been defined as the union of non-overlapping cells $\mathcal{C}_{i}^{e}$, as shown in Fig. 1(b). Consequently, the average on $\mathcal{C}_{i}$ can be obtained by summing Eq. (2.16) over the neighboring elements of $\mathcal{C}_{i}$, and separately, by summing Eq. (2.17) in a similar way. In other words, for all $e \in \mathcal{E}(i)$, Eqs. (2.16) and (2.17) are added to obtain, at the cell scale,

$$
\left\{\begin{aligned}
a_{i} \partial_{t} \bar{h}_{i}+\sum_{e \in \mathcal{E}(i)} \sum_{j \in \vartheta(i, e)} \boldsymbol{\phi}_{i j}^{h, e}= & \sum_{e \in \mathcal{E}(i)} \int_{\mathcal{C}_{i}^{e}}\left(\mathrm{~J} u^{\zeta}\right)_{\zeta=0} d \boldsymbol{\xi} \\
& -\sum_{e \in \mathcal{E}(i)} \int_{\partial \mathcal{C}_{i}^{e} \cap \partial e} \int_{0}^{h^{e}} \mathrm{JU} \cdot \widetilde{\boldsymbol{n}} d \zeta \widetilde{\boldsymbol{\ell}} \\
a_{i} \partial_{t}\left(\bar{h}_{i} \overline{\mathbf{U}}_{i}\right)+\sum_{e \in \mathcal{E}(i)} \sum_{j \in \vartheta(i, e)} \boldsymbol{\phi}_{i j}^{h \mathbf{U}, e}= & \sum_{e \in \mathcal{E}(i)} \int_{\mathcal{C}_{i}^{e}} \mathrm{~J}\left(\mathbf{U} u^{\zeta}+\underline{\mathbf{P}} \boldsymbol{g}^{\zeta}\right)_{\zeta=0} d \boldsymbol{\xi}-\sum_{e \in \mathcal{E}(i)} \int_{\mathcal{C}_{i}^{e}} \mathrm{~J}(\underline{\mathbf{P}} \mathrm{N})_{\zeta=h} d \boldsymbol{\xi} \\
& -\sum_{e \in \mathcal{E}(i)} \int_{\partial \mathcal{C}_{i}^{e} \cap \partial e} \int_{0}^{h^{e}} \mathrm{~J}(\mathbf{U} \otimes \mathbf{U}+\underline{\mathbf{P}}) \widetilde{\boldsymbol{n}} d \zeta \widetilde{d}
\end{aligned}\right.
$$


where we have defined

$$
a_{i} \bar{h}_{i}=\sum_{e \in \mathcal{E}(i)} a_{i}^{e} \bar{h}_{i}^{e} \quad \text { and } \quad a_{i} \bar{h}_{i} \overline{\mathbf{U}}_{i}=\sum_{e \in \mathcal{E}(i)} a_{i}^{e} \bar{h}_{i}^{e} \overline{\mathbf{U}}_{i}^{e},
$$

being $a_{i}=\sum_{e \in \vartheta(i)} a_{i}^{e}$ the total area of the cell $\mathcal{C}_{i}$. As already elaborated, the internal flux exchanges vanish at the cell scale (cf. Fig. 1), i.e.,

$$
\sum_{e \in \mathcal{E}(i)} \int_{\partial \mathcal{C}_{i}^{e} \cap \partial e} \int_{0}^{h^{e}} \mathrm{JU} \cdot \widetilde{\boldsymbol{n}} d \zeta \widetilde{d \ell}=0 \quad \text { and } \sum_{e \in \mathcal{E}(i)} \int_{\partial \mathcal{C}_{i}^{e} \cap \partial e} \int_{0}^{h^{e}} \mathrm{~J}(\mathbf{U} \otimes \mathbf{U}+\underline{\mathbf{P}}) \widetilde{\boldsymbol{n}} d \zeta \widetilde{d \ell}=0
$$

Therefore, the compact form of the cell scale discrete formulation (2.18) reads

$$
\begin{aligned}
a_{i} \frac{\partial}{\partial t} \overline{\mathbf{w}}_{i} & +\sum_{e \in \mathcal{E}(i)} \sum_{j \in \vartheta(i, e)} \int_{\partial \mathcal{C}_{i}^{e} \cap \partial \mathcal{C}_{j}^{e}} \int_{0}^{h^{e}} \widetilde{\mathbf{f}}(\mathbf{w}) \widetilde{\boldsymbol{n}} d \zeta \widetilde{d \ell} \\
& +\sum_{e \in \mathcal{E}(i)}\left(\int_{\mathcal{C}_{i}^{e}}\left(\begin{array}{c}
0 \\
\mathrm{~J} \underline{\mathbf{P}}_{\mathrm{N}}
\end{array}\right) d \boldsymbol{\xi}\right)_{\zeta=h}=\sum_{e \in \mathcal{E}(i)}\left(\int_{\mathcal{C}_{i}^{e}} \widetilde{\mathbf{f}}(\mathbf{w}) \boldsymbol{g}^{\zeta} d \boldsymbol{\xi}\right)_{\zeta=0},
\end{aligned}
$$

with

$$
\overline{\mathbf{w}}=\left(\begin{array}{c}
\bar{h} \\
\bar{h} \overline{\mathbf{U}}
\end{array}\right), \quad \mathbf{w}=\left(\begin{array}{c}
h \\
h \mathbf{U}
\end{array}\right), \quad \text { and } \quad \tilde{\mathbf{f}}(\mathbf{w})=\left(\begin{array}{c}
\mathbf{U}^{\mathrm{T}} \\
\mathbf{U} \otimes \mathbf{U}+\underline{\mathbf{P}}
\end{array}\right)
$$

In (2.20), $\mathbf{w}=\overline{\mathbf{w}}+\mathbf{w}^{\prime}$, where the fluctuation $\mathbf{w}^{\prime}$, with respect to a depth-averaged state $\overline{\mathbf{w}}$, is allowed for a general case, such as the shear shallow water flows modeled by $[48,49]$. In the present study, we concentrate on the numerical approach, so that only the depthaveraged state $\overline{\mathbf{w}}$ is considered at the current stage.

The discrete model (2.20) has been obtained under certain assumptions (for instance, each sub-cell is assumed to be flat). However, some of these assumptions can be relaxed without drastically changing the existing general formulation (2.20). At this moment, it is important to note that all the components of the velocity are unknown to the discrete model. The evolution of $\overline{\mathbf{w}}_{i}$ will be defined as soon as the fluxes $\boldsymbol{\phi}_{i}^{\mathbf{w}, e}$ or $\boldsymbol{\Phi}_{i j}^{\mathbf{F}, e}$ in (2.40) and the source term $\boldsymbol{\Phi}_{i}^{\mathbf{S}, e}$ in (2.40) are formulated as functions of the averaged values of the neighboring cell $\overline{\mathbf{w}}_{j}$.

Now the next task is to find a way to formulate the fluxes $\boldsymbol{\phi}_{i}^{\mathbf{w}, e}$ and the source terms as functions of the averaged values $\overline{\mathbf{w}}_{j}$. For a common interface between two adjacent cells, this can be achieved by the use of Riemann problems associated to an adapted reduced model. Other fluxes will be defined by means of the boundary conditions, particularly on the basal and free surfaces.

\subsection{Shallowness assumption, approximation associated to interactions with the flow surface \& fluxes $\phi_{i}^{\mathbf{w}, c}$}

The shallowness approximations can be applied when the typical flow thickness is much smaller than its extension tangential to the basal surface. In this context, the scaling anal- 
ysis helps to justify that the pressure is essentially dominated by the effect of hydrostatic forces and the curvature effect (centrifugal force) is of a slightly higher order when the basal surface is curved with a shallow curvature $[21,32,34,44]$. With the free surface assumption, i.e., vanishing pressure at the flow surface, one can determine the hydrostatic pressure by

$$
p \simeq-\rho_{\delta}(\zeta-h(t, \boldsymbol{\xi}))
$$

with $c=\boldsymbol{g}^{\zeta} \cdot \widehat{\boldsymbol{e}}_{z}$, when the curvature effect is neglected. However, the considered vertexcell consists of several triangular elements with variant normal directions, so that the cell is not flat. Hence, the curvature effect should be taken into account for the pressure as in $[21,32,34,44-46]$ and it reads

$$
p / \rho \simeq-\left(\kappa_{b}+g c\right)(\zeta-h(t, \boldsymbol{\xi})) \quad \text { with } \quad \kappa_{b}=\underline{\mathbf{C}}:(\underline{\mathbf{B}} \mathbf{U} \otimes \underline{\mathbf{B}} \mathbf{U}),
$$

where $\underline{\mathbf{C}}$ is an approximated curvature tensor of the vertex-centered cell and $\underline{\mathbf{B}}=\boldsymbol{g}^{\xi} \otimes \boldsymbol{g}_{\xi}+$ $\boldsymbol{g}^{\eta} \otimes \boldsymbol{g}_{\eta}$ is the projection on the tangent plane to the basal surface $[26,32,34]$. On the other hand, with the considered mapping, we locally have

$$
z-z_{0}=b(\boldsymbol{x})+c \zeta \quad \text { with } \quad b(\boldsymbol{x})=b(\boldsymbol{x}(\boldsymbol{\xi}))=b_{i}-z_{0}+\widetilde{z}(\boldsymbol{\xi}),
$$

where $\widetilde{z}(\boldsymbol{\xi})=\xi \boldsymbol{g}_{\xi} \cdot \widehat{\boldsymbol{e}}_{z}+\eta \boldsymbol{g}_{\eta} \cdot \widehat{\boldsymbol{e}}_{z}$. The generalized stress tensor $\underline{\mathbf{P}}=\left(p / \rho+g\left(z-z_{0}\right)\right) \underline{\mathbf{I}}-\underline{\boldsymbol{\tau}} / \rho$ is then approximated as

$$
\underline{\mathbf{P}} \simeq\left(g(c h+b)-\kappa_{b}(\zeta-h)\right) \underline{\mathbf{I}}-\underline{\boldsymbol{\tau}} / \rho .
$$

At the flow surface $(\zeta=h)$, the stress tensor $(2.22)$ reads

$$
(\underline{\mathbf{P}})_{\zeta=h} \simeq g(c h+b) \underline{\mathbf{I}}-(\underline{\boldsymbol{\tau}})_{\zeta=h} / \rho
$$

Then, the component of the stress flux associated to the free surface isotropic pressure $\rho g(c h+b)$ is reformulated to introduce a divergence form. Indeed,

$$
g(c h+b)\left(\boldsymbol{g}^{\xi} \partial_{\xi} h+\boldsymbol{g}^{\eta} \partial_{\eta} h\right)=\boldsymbol{g}^{\xi} \partial_{\xi}\left(g c \frac{h^{2}}{2}+g b h\right)+\boldsymbol{g}^{\eta} \partial_{\eta}\left(g c \frac{h^{2}}{2}+g b h\right)-g h \nabla_{\widetilde{\boldsymbol{x}}} b,
$$

where straightforward algebra gives $\nabla_{\widetilde{\boldsymbol{x}}} b=\boldsymbol{g}^{\tilde{\xi}} \partial_{\tilde{\xi}} \widetilde{z}+\boldsymbol{g}^{\eta} \partial_{\eta} \widetilde{z}$ with $\widetilde{z}(\boldsymbol{\xi})=\xi \boldsymbol{g}_{\xi} \cdot \widehat{\boldsymbol{e}}_{z}+\eta \boldsymbol{g}_{\eta} \cdot \widehat{\boldsymbol{e}}_{z}$. Therefore, as $\mathrm{N}=-\boldsymbol{g}^{\tilde{\zeta}} \partial_{\xi} h-\boldsymbol{g}^{\eta} \partial_{\eta} h+\boldsymbol{g}^{\zeta}$, we get

$$
\begin{aligned}
(\underline{\mathbf{P}} \mathrm{N})_{\zeta=h}= & -\boldsymbol{g}^{\tau} \partial_{\tilde{\zeta}}\left(g c \frac{h^{2}}{2}+g b h\right)-\boldsymbol{g}^{\eta} \partial_{\eta}\left(g c \frac{h^{2}}{2}+g b h\right) \\
& +g h \nabla_{\widetilde{\boldsymbol{x}}} b+g(c h+b) \boldsymbol{g}^{\zeta}-\frac{1}{\rho}(\underline{\boldsymbol{\tau}} \mathrm{N})_{\zeta=h} .
\end{aligned}
$$


Locally on a triangular element, J, $c, g^{\tau}$ and $g^{\eta}$ are constant; thus, the third term on the left-hand side (LHS) of (2.20) is recast into

$$
\begin{aligned}
& \int_{\mathcal{C}_{i}^{e}}\left(\begin{array}{c}
0 \\
\mathrm{~J} \underline{\mathbf{P} N}
\end{array}\right)_{\zeta=h} d \boldsymbol{\xi}=-\sum_{j \in \vartheta(i, e)} \int_{\partial \mathcal{C}_{i}^{e} \cap \partial \mathcal{C}_{j}^{e}}\left(\mathrm{~J}\left(g c \frac{h^{2}}{2}+g b h\right) \underline{\mathbf{I}}\right) \tilde{\boldsymbol{n}} \tilde{d \ell} \\
& -\int_{\partial \mathcal{C}_{i}^{e} \cap \partial e}\left(\mathrm{~J}\left(g c{\frac{h^{2}}{2}}^{0} g b h\right) \underline{\mathbf{I}}\right) \widetilde{\boldsymbol{n}} \widetilde{d}+\int_{\mathcal{C}_{i}^{e}}\left(\begin{array}{c}
0 \\
\operatorname{Jgh} \nabla_{\widetilde{\boldsymbol{x}}} b
\end{array}\right) d \tilde{\boldsymbol{\zeta}} \\
& +\int_{\mathcal{C}_{i}^{e}}\left(\begin{array}{c}
0 \\
\mathrm{~J} g(c h+b) \boldsymbol{g}^{\zeta}
\end{array}\right) d \boldsymbol{\zeta}-\int_{\mathcal{C}_{i}^{e}}\left(\begin{array}{c}
0 \\
\frac{1}{\rho} \mathrm{J} \underline{\mathrm{N}}
\end{array}\right)_{\zeta=h} d \boldsymbol{\zeta} .
\end{aligned}
$$

Here, we have used the fact that the normal vector is constant on the interface $\partial \mathcal{V}_{i}^{e} \cap \partial \mathcal{V}_{j}^{e}$ that contains $\partial \mathcal{C}_{i}^{e} \cap \partial \mathcal{C}_{j}^{e}$. By using this decomposition, we artificially separate the contribution of the free flow surface into several components. The first integral on the right-hand side (RHS) of (2.24) is a lateral flow in the hyperbolic framework. The third to the last integrals on the RHS will be considered as source terms, where the coherence of all of these different approximations is for the issue of well-balanced schemes [47]. The second integral is associated to interactions within the cell, so that it will be canceled out at the cell level and no additional treatment is needed at this scale.

In viewing (2.20) and (2.24), the total flux associated to the interface $\partial \mathcal{C}_{i}^{e} \cap \partial \mathcal{C}_{j}^{e}$ now reads

$$
\boldsymbol{\phi}_{i j}^{\mathbf{w}, e}=\int_{\partial \mathcal{C}_{i}^{e} \cap \partial \mathcal{C}_{j}^{e}} \int_{0}^{h} \widetilde{J} \tilde{\mathbf{f}}(\mathbf{w}) \widetilde{\boldsymbol{n}} d \zeta \tilde{d \ell}-\int_{\partial \mathcal{C}_{i}^{e} \cap \partial \mathcal{C}_{j}^{e}}\left(\mathrm{~J}\left(g c \frac{h^{2}}{2}+g b h\right) \underline{\mathbf{I}}\right) \widetilde{\boldsymbol{n}} \widetilde{\boldsymbol{\ell}}
$$

With $\widetilde{\mathbf{f}}$ defined in $(2.20)$ and $\underline{\mathbf{P}} \simeq g(c h+b) \underline{\mathbf{I}}-\underline{\boldsymbol{\tau}} / \rho-\kappa_{b}(\zeta-h) \underline{\mathbf{I}}$, the first integral in (2.25) can be approximated by

$$
\begin{aligned}
& \int_{\partial \mathcal{C}_{i}^{e} \cap \partial \mathcal{C}_{j}^{e}} \int_{0}^{h} \widetilde{\mathbf{f}}(\mathbf{w}) \widetilde{\boldsymbol{n}} d \zeta d \widetilde{\ell} \simeq \int_{\partial \mathcal{C}_{i}^{e} \cap \partial \mathcal{C}_{j}^{e}} \mathrm{Jh \widetilde { \mathbf {f } }}(\mathbf{w}) \widetilde{\boldsymbol{n}} d \widetilde{\ell}
\end{aligned}
$$

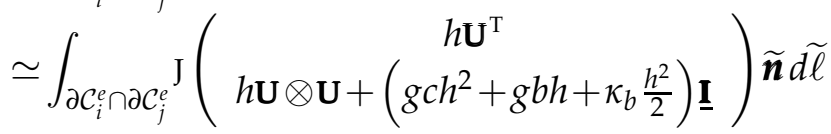

$$
\begin{aligned}
& -\int_{\partial \mathcal{C}_{i}^{e} \cap \partial \mathcal{C}_{j}^{e}}\left(\begin{array}{c}
0 \\
\frac{1}{\rho} \mathrm{J} h \underline{\boldsymbol{\tau}} \widetilde{\boldsymbol{n}}
\end{array}\right) d \widetilde{\ell},
\end{aligned}
$$

for a uniform velocity distribution along the flow thickness. The flux $\boldsymbol{\phi}_{i j}^{\mathbf{w}, e}$ in (2.25) then can be decomposed into a hyperbolic component $\boldsymbol{\Phi}_{i j}^{\mathrm{F}, e}$ and a viscous/frictional component $\boldsymbol{\Phi}_{i j}^{\underline{\tau}}$, , , i.e.,

$$
\boldsymbol{\phi}_{i j}^{\mathbf{w}, e} \simeq \boldsymbol{\Phi}_{i j}^{\mathbf{F}, e}-\boldsymbol{\Phi}_{i j}^{\boldsymbol{\tau}, e}
$$


with

$$
\boldsymbol{\Phi}_{i j}^{\mathbf{F}, e}=\int_{\partial C_{i}^{e} \cap \partial \mathcal{C}_{j}^{e}} \boldsymbol{\Phi}^{e}(\mathrm{~J} \widetilde{\boldsymbol{n}}, \mathbf{w}) \tilde{d \ell} \quad \text { and } \quad \boldsymbol{\Phi}_{i j}^{\underline{\underline{\tau}} e}=\int_{\partial C_{i}^{e} \cap \partial C_{j}^{e}} \mathrm{~J}\left(\begin{array}{c}
0 \\
\frac{1}{\rho} \underline{\boldsymbol{\tau}} \widetilde{\boldsymbol{n}}
\end{array}\right) d \zeta \tilde{d \ell} .
$$

In (2.27), $\boldsymbol{\Phi}^{e}(\widetilde{\boldsymbol{n}}, \mathbf{w})$ denotes the normal flux at the interface and it is given by

$$
\boldsymbol{\Phi}^{e}(\mathrm{~J} \widetilde{\boldsymbol{n}}, \mathbf{w})=\left(\begin{array}{c}
\mathrm{J} h \mathrm{U} \cdot \widetilde{\boldsymbol{n}} \\
\mathrm{J} h \mathrm{U}(\mathrm{U} \cdot \widetilde{\boldsymbol{n}})+\mathrm{J}\left(g c+\kappa_{b}\right) \frac{h^{2}}{2} \widetilde{\boldsymbol{n}}
\end{array}\right)=\left(J \widetilde{\boldsymbol{n}} \cdot \boldsymbol{g}_{\xi}\right) \mathbf{F}(\mathbf{w})+\left(J \widetilde{\boldsymbol{n}} \cdot \boldsymbol{g}_{\eta}\right) \mathbf{G}(\mathbf{w}),
$$

where $\mathbf{w}=(h, h \mathbf{U})^{\mathrm{T}}, \mathbf{F} \equiv \mathbf{F}(\mathbf{w})$ and $\mathbf{G} \equiv \mathbf{G}(\mathbf{w})$, with

$$
\mathbf{F}=\left(\begin{array}{c}
h u^{\xi} \\
h \mathbf{U} u^{\xi}+\left(g c+\kappa_{b}\right) \frac{h^{2}}{2} \boldsymbol{g}^{\xi}
\end{array}\right) \quad \text { and } \quad \mathbf{G}=\left(\begin{array}{c}
h u^{\eta} \\
h \mathbf{U} u^{\eta}+\left(g c+\kappa_{b}\right) \frac{h^{2}}{2} \boldsymbol{g}^{\eta}
\end{array}\right) .
$$

Note that the vectors in the previous equations are still three-dimensional quantities even after the depth-averaging process; for example, the velocity field $\mathbf{U}$ is still given as in its definition (2.6). Indeed, our basal surface is still in 3D form and has not been reduced to $2 \mathrm{D}$, as is usual for flat planes.

\subsection{Riemann problem \& flux $\Phi_{i j}^{\mathrm{F}, e}$ at the interface scale}

We proceed to describe how the numerical flux $\boldsymbol{\Phi}_{i j}^{e}$ is computed as a function of the intermediate state $\mathbf{w}_{i j}^{e}$, solution of an appropriate Riemann problem. The flux $\Phi^{e}(\boldsymbol{n}, \mathbf{w})$ is a linear function of $\boldsymbol{n}$. Assuming that the approximated state $\boldsymbol{w}_{i j}^{e}$ does not vary along the interface, we have

$$
\boldsymbol{\Phi}_{i j}^{\mathbf{F}, e}=\boldsymbol{\Phi}^{e}\left(\mathrm{~N}_{i j}^{e}, \mathbf{w}_{i j}^{e}\right), \quad \text { with } \quad \mathrm{N}_{i j}^{e}=\int_{\partial \mathcal{C}_{i}^{e} \cap \partial \mathcal{C}_{j}^{e}} \mathrm{~J} \widetilde{\boldsymbol{n}} \tilde{\mathrm{\ell}}
$$

Refer to Appendix A.2 for the definitions of the cell interface normal vectors $\mathrm{N}_{i j}^{e}$ on a flat triangular element. Now, in order to obtain an approximation of the above flux, let us consider the following Riemann problem at the interface scale:

$$
\frac{\partial}{\partial t} \mathbf{w}+\frac{\partial}{\partial n} \boldsymbol{\Phi}^{e}(\boldsymbol{n}, \mathbf{w})=0 \quad \text { with } \quad \mathbf{w}(t=0, n)= \begin{cases}\mathbf{w}_{l}, & \text { if } n<0 \\ \mathbf{w}_{r}, & \text { if } n>0\end{cases}
$$

where $n$ is the coordinate associated to the direction $n$. This system is hyperbolic and the eigenvalues (assuming $\kappa_{b}$ constant) are

$$
\lambda=\mathbf{U} \cdot \boldsymbol{n}, \quad \lambda=\mathbf{U} \cdot \boldsymbol{n}-\sqrt{\left(g c+\kappa_{b}\right) h} \quad \text { and } \quad \lambda=\mathbf{U} \cdot \boldsymbol{n}+\sqrt{\left(g c+\kappa_{b}\right) h} .
$$


Then, an approximate Riemann solver will define the flux at $n=0$ as a function of the states $\mathbf{w}_{l}$ and $\mathbf{w}_{r}$, e.g.,

$$
\widetilde{\boldsymbol{\Phi}}^{e}\left(\boldsymbol{n}, \mathbf{w}_{l}, \mathbf{w}_{r}\right)=\frac{1}{2}\left[\boldsymbol{\Phi}\left(\boldsymbol{n}, \mathbf{w}_{l}\right)+\boldsymbol{\Phi}\left(\boldsymbol{n}, \mathbf{w}_{r}\right)+|\mathcal{A}|\left(\mathbf{w}_{l}-\mathbf{w}_{r}\right)\right],
$$

being $\mathcal{A} \simeq \frac{\partial}{\partial \mathbf{w}} \boldsymbol{\Phi}(\boldsymbol{n}, \mathbf{w})$ an approximated Jacobian. For this form, one of the simplest approximations is the so-called Rusanov solver that considers $|\mathcal{A}|=\left(\max _{\mathbf{w}}|\lambda(\mathbf{w})|\right) \underline{\mathbf{I}}$. For an HLL-type scheme, the numerical flux takes the following simplified form instead:

$$
\widetilde{\boldsymbol{\Phi}}^{e}\left(\boldsymbol{n}, \mathbf{w}_{l}, \mathbf{w}_{r}\right)=\frac{S_{r} \boldsymbol{\Phi}\left(\boldsymbol{n}, \mathbf{w}_{l}\right)-S_{l} \boldsymbol{\Phi}\left(\boldsymbol{n}, \mathbf{w}_{r}\right)-S_{l} S_{r}\left(\mathbf{w}_{l}-\mathbf{w}_{r}\right)}{S_{r}-S_{l}},
$$

where $S_{l}$ and $S_{r}$ are the maximum wave speeds traveling to the left and right, respectively. For the first order approximation, the numerical flux is estimated as

$$
\boldsymbol{\Phi}_{i j}^{\mathbf{F}, e} \simeq \widetilde{\boldsymbol{\Phi}}^{e}\left(\mathrm{~N}_{i j}^{e}, \overline{\mathbf{w}}_{i}, \overline{\mathbf{w}}_{j}\right) .
$$

This approximation of the flux is conservative, i.e., the outward normal vector associated to the cell $\mathcal{C}_{j}$ at the interface with $\mathcal{C}_{i}$ will be opposite to $\mathrm{N}_{i j}^{e}$. In other words, $\mathrm{N}_{j i}^{e}=-\mathrm{N}_{i j}^{e}$, and by simple algebraic manipulations, one can show that $\widetilde{\boldsymbol{\Phi}}^{e}\left(\mathrm{~N}_{j i}^{e}, \overline{\mathbf{w}}_{j}, \overline{\mathbf{w}}_{i}\right)=-\widetilde{\boldsymbol{\Phi}}^{e}\left(\mathrm{~N}_{i j}^{e}, \overline{\mathbf{w}}_{i}, \overline{\mathbf{w}}_{j}\right)$.

Let us mention that the current flux is obtained under the strong assumption that, locally at the interface, the fluctuating state $\mathbf{w}$ is constant in the thin layer and acts like the mean value $\overline{\mathbf{w}}$. In order to improve the approximation of the interface flux, interpolations are often employed, via the MUSCL approach. The MUSCL reconstruction is a purely geometrical interpolation. It is also possible to proceed with certain physical interpolations that take into account the thin profile of the local flow, that is,

$$
\boldsymbol{\Phi}_{i j}^{\mathbf{F}, e} \simeq \frac{1}{h_{i j}} \int_{0}^{h_{i j}} \widetilde{\boldsymbol{\Phi}}^{e}\left(\mathrm{~N}_{i j}^{e}, \mathbf{w}_{i}\left(\boldsymbol{\zeta}_{i j}, \zeta\right), \mathbf{w}_{j}\left(\boldsymbol{\zeta}_{i j}, \zeta\right)\right) d \zeta,
$$

where $\mathbf{w}_{i}\left(\boldsymbol{\zeta}_{i j}, \zeta\right)=\overline{\mathbf{w}}_{i}+\mathbf{w}_{i}^{\prime}\left(\boldsymbol{\xi}_{i j}, \zeta\right)$ and $\mathbf{w}_{j}\left(\boldsymbol{\zeta}_{i j}, \zeta\right)=\overline{\mathbf{w}}_{j}+\mathbf{w}_{j}^{\prime}\left(\boldsymbol{\xi}_{i j}, \zeta\right)$. The interface profiles defined by $\mathbf{w}_{i}^{\prime}$ and $\mathbf{w}_{j}^{\prime}$ must be built under physical considerations. This profiling is inevitable for strongly sheared flows. For example, in [48-51] it is proposed to introduce new variables based on the evolution of the kinetic energy, in order to simplify this construction.

With respect to the cell-scale approximation (2.20), we find it convenient to review what we have so far:

$$
\begin{aligned}
a_{i} \frac{\partial}{\partial t} \overline{\mathbf{w}}_{i}+\sum_{e \in \mathcal{E}(i)} \sum_{j \in \vartheta(i, e)} \boldsymbol{\Phi}_{i j}^{\mathbf{F}, e}= & \sum_{e \in \mathcal{E}(i)} \sum_{j \in \vartheta(i, e)} \boldsymbol{\Phi}_{i j}^{\underline{\tau}, e}+\sum_{e \in \mathcal{E}(i)}\left(\int_{\mathcal{C}_{i}^{e}} \widetilde{\widetilde{f}}(\mathbf{w}) \boldsymbol{g}^{\zeta} d \boldsymbol{\xi}\right)_{\zeta=0} \\
& +\sum_{e \in \mathcal{E}(i)} \int_{\mathcal{C}_{i}^{e}}\left(\begin{array}{c}
0 \\
\mathrm{~J} \underline{\mathrm{T}} \mathrm{N} / \rho-\mathrm{J} g(c h+b) \boldsymbol{g}^{\zeta}-\mathrm{J} g h \nabla_{\widetilde{\boldsymbol{x}}} b
\end{array}\right)_{\zeta=h} d \boldsymbol{\xi} .
\end{aligned}
$$

For constructing the numerical scheme, we still need to provide the approximation for the fluxes and pressure forces on the basal surface, and the viscous/frictional fluxes. 


\subsection{Source term $\Phi_{i}^{\mathrm{S}, e} \&$ approximation associated to interactions with the basal surface}

We assume here that the basal surface is impermeable, so that the velocity component normal to the bottom is zero, i.e.,

$$
u_{b}^{\zeta} \equiv 0
$$

For ease of description, the drag force equilibrium at the basal surface is assumed to be

$$
\underline{\mathbf{P}}_{b} \boldsymbol{g}^{\zeta}=g b \boldsymbol{g}^{\zeta}+\frac{1}{\rho}\left(p_{b}-\boldsymbol{g}^{\zeta} \cdot \underline{\boldsymbol{\tau}}_{b} \boldsymbol{g}^{\zeta}\right) \boldsymbol{g}^{\zeta}-\frac{1}{\rho} \mu \tilde{p}_{b} \frac{\mathrm{U}}{\|\mathrm{U}\|},
$$

where the last term represents the Coulomb friction law (see, for instance, $[1,18]$ ), with $\mu$ being the friction coefficient and $\tilde{p}_{b}=\left(p_{b}-\boldsymbol{g}^{\zeta} \cdot \underline{\boldsymbol{\tau}}_{b} \boldsymbol{g}^{\zeta}\right)^{+}$the positive part of the normal stress on the basal surface. The friction coefficient $\mu$ can be interpreted as the ratio between shear and normal stresses; $\mu \simeq\|\underline{\tau}\| /|p|$, see e.g., [52,53].

In the context of long-wave laminar flows, the shear stress normal to the basal surface is asymptotically negligible compared to $p_{b}$, i.e., $p_{b}-\boldsymbol{g}^{\zeta} \cdot \underline{\boldsymbol{\tau}}_{b} \boldsymbol{g}^{\zeta} \simeq p_{b}$, so that the approximate basal pressure is $p_{b} \simeq \rho\left(g c+\kappa_{b}\right) h$, and we arrive at

$$
\begin{aligned}
& \left(\int_{\mathcal{C}_{i}^{e}} \widetilde{\mathbf{f}}(\mathbf{w}) \boldsymbol{g}^{\zeta} d \boldsymbol{\zeta}\right)_{\zeta=0}=\int_{\mathcal{C}_{i}^{e}} \mathrm{~J}\left(\begin{array}{c}
0 \\
\underline{\mathbf{P}}_{b} \boldsymbol{g}^{\zeta}
\end{array}\right) d \boldsymbol{\zeta} \\
& \left.\simeq \int_{\mathcal{C}_{i}^{e}}\left(\begin{array}{c}
0 \\
\mathrm{~J}
\end{array} g(c h+b)+\kappa_{b} h\right] \boldsymbol{g}^{\zeta}\right) d \boldsymbol{\zeta}-\int_{\mathcal{C}_{i}^{e}}\left(\begin{array}{c}
0 \\
\mathrm{~J} \mu \frac{p_{b}}{\rho} \frac{\mathbf{U}}{\|\mathbf{U}\|}
\end{array}\right) d \boldsymbol{\xi} .
\end{aligned}
$$

In (2.33), the first term on the RHS denotes the force acting normal to the bottom; the second one stands for a force tangent to the basal surface. Therefore, together with the last two terms in (2.31), we obtain the source term and it reads

$$
\begin{aligned}
\boldsymbol{\Phi}_{i}^{\mathbf{S}, e} & =\left(\int_{\mathcal{C}_{i}^{e}} \widetilde{\mathbf{f}}(\mathbf{w}) \boldsymbol{g}^{\zeta} d \boldsymbol{\zeta}\right)_{\zeta=0}+\int_{\mathcal{C}_{i}^{e}}\left(\begin{array}{c}
0 \\
\mathrm{~J} \underline{\boldsymbol{\tau}} / \rho-\mathrm{J} g(c h+b) \boldsymbol{g}^{\zeta}-\mathrm{J} g h \nabla_{\widetilde{\boldsymbol{x}}} b
\end{array}\right)_{\zeta=h} d \boldsymbol{\xi} \\
& \simeq \int_{\mathcal{C}_{i}^{e}} \mathrm{~J} \mathbf{S} d \boldsymbol{\zeta},
\end{aligned}
$$

where

$$
\mathbf{S}=\mathbf{S}(\mathbf{w})=\left(\frac{1}{\rho}(\underline{\boldsymbol{\tau}} \mathrm{N})_{\zeta=h}-g h\left(\nabla_{\widetilde{\boldsymbol{x}}} b+c \mu \frac{\mathbf{U}}{\|\mathbf{U}\|}\right)-\kappa_{b} h\left(\mu \frac{\mathbf{U}}{\|\mathbf{U}\|}-\mathbf{g}^{\zeta}\right)\right) .
$$

As the drag free condition at the flow surface is widely applied, one has $(\underline{\boldsymbol{\tau}} \mathrm{N})_{\zeta=h} \simeq 0$, and this source term recovers the one proposed in [24, equation (2.49)]. In (2.35), $\kappa_{b}=\underline{\mathbf{C}}$ : $(\underline{\mathbf{B}} \mathbf{U} \otimes \underline{\mathbf{B U}})$ represents the curvature contribution with the curvature tensor

$$
\underline{\mathbf{c}} \equiv\left(c^{2} \underline{\mathbf{I}}+\boldsymbol{g}^{\zeta} \otimes \boldsymbol{g}^{\zeta}\right)\left(\partial_{\widetilde{x}} \boldsymbol{g}^{\zeta}\right)
$$


Here we recall $\boldsymbol{g}^{\zeta}=(-\boldsymbol{s}, c)^{\mathrm{T}}$ is the normal to the basal surface.

Remark 2.1. The approximation of $g h \nabla_{\widetilde{x}} b$ relies on the approach proposed in $[47,54]$ and is based on the hydrostatic reconstructed water depth.

The curvature in (2.36) is to be approximated for a vertex-centered cell, which is composed by several triangular elements (cf. Fig. 1(b)). Since each triangular element has its own normal vector $\boldsymbol{g}^{\zeta}$, the curvature can be computed either by a polyhedral approximation [55] or by the general approach suggested in [46]. In the present study, we follow the strategy proposed in [55], that the tensor of curvature, at a vertex $i$, is estimated as

$$
\underline{\mathbf{c}}_{i} \simeq \sum_{j \in \vartheta(i)} \omega_{i j} \mathcal{H}_{i j} \mathbf{B}_{i j} \otimes \mathbf{B}_{i j}
$$

where $\omega_{i j}$ is a positive weight proportional to the surface area of the triangle element $j$ within the cell $i$, such that $\sum_{j \in \vartheta(i)} \omega_{i j}=1$. In addition,

$$
\mathbf{B}_{i j}=\frac{\left(\underline{\mathbf{I}}-\overline{\boldsymbol{g}_{i}^{\zeta}} \otimes \overline{\boldsymbol{g}_{i}^{\zeta}}\right)\left(\widetilde{\boldsymbol{x}}_{i}-\widetilde{\boldsymbol{x}}_{j}\right)}{\left\|\left(\underline{\mathbf{I}}-\overline{\boldsymbol{g}_{i}^{\zeta}} \otimes \overline{\boldsymbol{g}_{i}^{\zeta}}\right)\left(\widetilde{\boldsymbol{x}}_{i}-\widetilde{\boldsymbol{x}}_{j}\right)\right\|} \quad \text { and } \quad \mathcal{H}_{i j}=\frac{2 \overline{\boldsymbol{g}_{i}^{\zeta}} \cdot\left(\widetilde{\boldsymbol{x}}_{i}-\widetilde{\boldsymbol{x}}_{j}\right)}{\left\|\widetilde{\boldsymbol{x}}_{i}-\widetilde{\boldsymbol{x}}_{j}\right\|^{2}} \text {, with } \overline{\boldsymbol{g}_{i}^{\zeta}}=\frac{\sum_{e \in \vartheta(i)} a_{i}^{e}\left(\boldsymbol{g}^{\zeta}\right)_{e}}{\left\|\sum_{e \in \vartheta(i)} a_{i}^{e}\left(\boldsymbol{g}^{\zeta}\right)_{e}\right\|} \text {. }
$$

\subsection{Final scheme for weakly sheared flows}

With the source term (2.34), the cell-scale approximation (2.31) then reads

$$
a_{i} \frac{\partial}{\partial t} \overline{\mathbf{w}}_{i}+\sum_{e \in \mathcal{E}(i)} \sum_{j \in \vartheta(i, e)} \boldsymbol{\Phi}_{i j}^{\mathbf{F}, e}=\sum_{e \in \mathcal{E}(i)} \sum_{j \in \vartheta(i, e)} \boldsymbol{\Phi}_{i j}^{\underline{\tau}, e}+\sum_{e \in \mathcal{E}(i)} \boldsymbol{\Phi}_{i}^{\mathbf{s}, e} \quad \text { with } \quad \overline{\mathbf{w}}_{i}=\left(\begin{array}{c}
\bar{h}_{i} \\
\bar{h}_{i} \overline{\mathbf{U}}_{i}
\end{array}\right),
$$

where $\overline{\mathbf{w}}_{i}$ is the mean value within the vertex-centered cell $\mathcal{E}(i)$. The viscous flux $\boldsymbol{\Phi}, \boldsymbol{\tau}_{i j}^{\boldsymbol{\tau}}$, , the first term on the RHS of (2.38) and defined in (2.27), represents the viscous friction from the neighboring cells. It is generally of higher order in scaling analysis and neglected in the resultant model equations $[1-3,6,17,18,21]$, so that we have the equation system at the cell-scale,

$$
a_{i} \frac{\partial}{\partial t} \overline{\mathbf{w}}_{i}+\sum_{e \in \mathcal{E}(i)} \sum_{j \in \vartheta(i, e)} \boldsymbol{\Phi}_{i j}^{\mathbf{F}, e}=\sum_{e \in \mathcal{E}(i)} \boldsymbol{\Phi}_{i}^{\mathbf{S}, e} \quad \text { with } \quad a_{i} \overline{\mathbf{w}}_{i}=\sum_{e \in \mathcal{E}(i)} a_{i}^{e} \overline{\mathbf{w}}_{i}^{e} .
$$

For rigid basal surfaces, where neither erosion nor deposition takes place, the area of the triangular element remains invariant, i.e., $a_{i}^{e}=$ const. By ignoring the internal fluxes within the cell, that will cancel out at the cell scale, we arrive at the approach at the element-scale, 


$$
a_{i}^{e} \frac{\partial}{\partial t} \overline{\mathbf{w}}_{i}^{e}+\sum_{j \in \vartheta(i, e)} \boldsymbol{\Phi}_{i j}^{\mathbf{F}, e}=\boldsymbol{\Phi}_{i}^{\mathbf{S}, e}
$$

where the flux $\boldsymbol{\Phi}_{i j}^{\mathbf{F}, e}$ is given in (2.27) and the source $\boldsymbol{\Phi}_{i}^{\mathbf{S}, e}$ is defined in (2.34). Eq. (2.40) can be viewed as a weak formulation locally associated to the following system:

$$
\frac{\partial}{\partial t}(\mathrm{~J} \overline{\mathbf{w}})+\frac{\partial}{\partial \xi}(\mathrm{J} \mathbf{F}(\overline{\mathbf{w}}))+\frac{\partial}{\partial \eta}(\mathrm{JG}(\overline{\mathbf{w}}))=\mathrm{JS}(\overline{\mathbf{w}}),
$$

where F, G and S are defined and given in (2.28) and (2.35), respectively. The equation system (2.41), with (2.28) and (2.35), is similar to the ones proposed in Bouchut and Westdickenberg [24], Luca et al. [26] or Tai et al. [34], which are single-phase models for shallow flows over complex topographies. However, in this context, system (2.40) only describes a local behavior and there is no need to define a nonsingular global mapping. Despite this, the proposed multi-mesh-scale approach is still constrained by the limitation of a "shallow curvature", as it inherits the scaling analysis from the models for arbitrary topographies $[24,26,32,34]$.

\section{Numerical examples}

In this section, we propose several test cases for validation of the proposed approach, using quasi-1D flows on flat planes. In Section 4, the numerical strategy will be applied on a complex topography.

We always use 2D meshes, even for quasi-1D flows. For all the simulations performed here, MUSCL reconstruction is used to achieve second order accuracy for smooth solutions. For non-smooth solutions, slope limiters (minmod and van Albada) are used to guarantee that a maximum principle is satisfied at the discrete level: the total variation is diminishing (TVD). The stability condition of the explicit scheme is obtained by local estimations of the maximum wave speed $\left(\lambda_{e}\right)$ and the minimum mesh length scale $\left(\delta \ell_{e}\right)$. Therefore, the time step $\Delta t$ is computed as

$$
\Delta t=\mathrm{CFL} * \min _{e}\left(\frac{\delta \ell_{e}}{\lambda_{e}}\right) .
$$

In practice, according to the estimation used for different meshes, a CFL number of 0.8 always achieved numerical stability of the explicit scheme.

\subsection{Quasi-1D flows on horizontal flat planes: Riemann problems}

The Riemann problem (also known as the dam break problem in hydraulics) is an initial value problem where a stationary flow body is blocked in a channel and is set in motion 
by suddenly removing the vertical blocking plate. In this section, we consider a rectangular domain $\Omega=\left[0, x_{L}\right] \times\left[0, y_{L}\right]$ and a "structured" triangular mesh of $169 * 57$ vertices and 11816 triangular elements. Then the initial condition is

$$
h(x, y)=\left\{\begin{array}{ll}
h_{L} & \text { for } 0 \leq x \leq x_{0}, \\
h_{R} & \text { for } x_{0}<x \leq x_{L},
\end{array} \text { and } u(x)=0.0 \text { for } 0 \leq x \leq x_{L} .\right.
$$

In (3.1), $x_{0}=1.6 \mathrm{~m}$ and $x_{L}=4.2 \mathrm{~m}$. The transverse length is $y_{L}=1.4 \mathrm{~m}$. The basal surface is defined by $b(x, y)=0$ and there is no basal friction. The boundary conditions are non reflecting in the $x$-direction and periodic in the $y$-direction.

\section{Dam break flow: Ritter's exact solution}

The initial condition for the Ritter's test is defined with

$$
h_{L}=1.0 \mathrm{~m} \quad \text { and } \quad h_{R}=0.0 \mathrm{~m} \text {. }
$$

This is an initial condition with a dry-land on the right-hand side. The exact solution is composed of a left going rarefaction wave that is a concave parabola connecting the constant water depth $h_{L}$ and the dry zone $\left(h_{R}=0\right)$. This parabola covers the range $\left[x_{A}(t), x_{B}(t)\right]$, extending with $x_{A}(t)=x_{0}-t \sqrt{g h_{L}}$ and $x_{B}(t)=x_{0}+2 t \sqrt{g h_{L}}$. Hence, the evolution of the depth's distribution reads (e.g. [56] or [57])

$$
h(x, y, t)= \begin{cases}h_{L} & \text { for } x \leq x_{A}(t) \\ \frac{4}{9 g}\left(\sqrt{g h_{L}}-\frac{x-x_{0}}{2 t}\right)^{2} & \text { for } x_{A}(t)<x<x_{B}(t), \\ 0.0 & \text { for } x_{B}(t) \leq x\end{cases}
$$

for the flow depth, where the corresponding velocity is linearly distributed in the rarefaction

$$
u(x, y, t)= \begin{cases}0.0 & \text { for } x \leq x_{A}(t) \\ \frac{2}{3}\left(\sqrt{g h_{L}}+\frac{x-x_{0}}{t}\right) & \text { for } x_{A}(t)<x<x_{B}(t), \\ 0.0 & \text { for } x_{B}(t) \leq x\end{cases}
$$

Here we recall that $h(x, y, t)$ is uniformly distributed in the lateral $y$-direction. The computed depth distributions are respectively shown in Fig. 5 at $t=0.0,0.225$ and $0.45 \mathrm{~s}$, where the corresponding section views along the central line $(y=0.7)$ are shown in the right panels. In the right panels of Fig. 5 , the blue " $x$ " markers denote the computed results and the analytical solutions are depicted by red lines. The computed results demonstrate the capability of the present approach of adequately describing this dam break problem. 

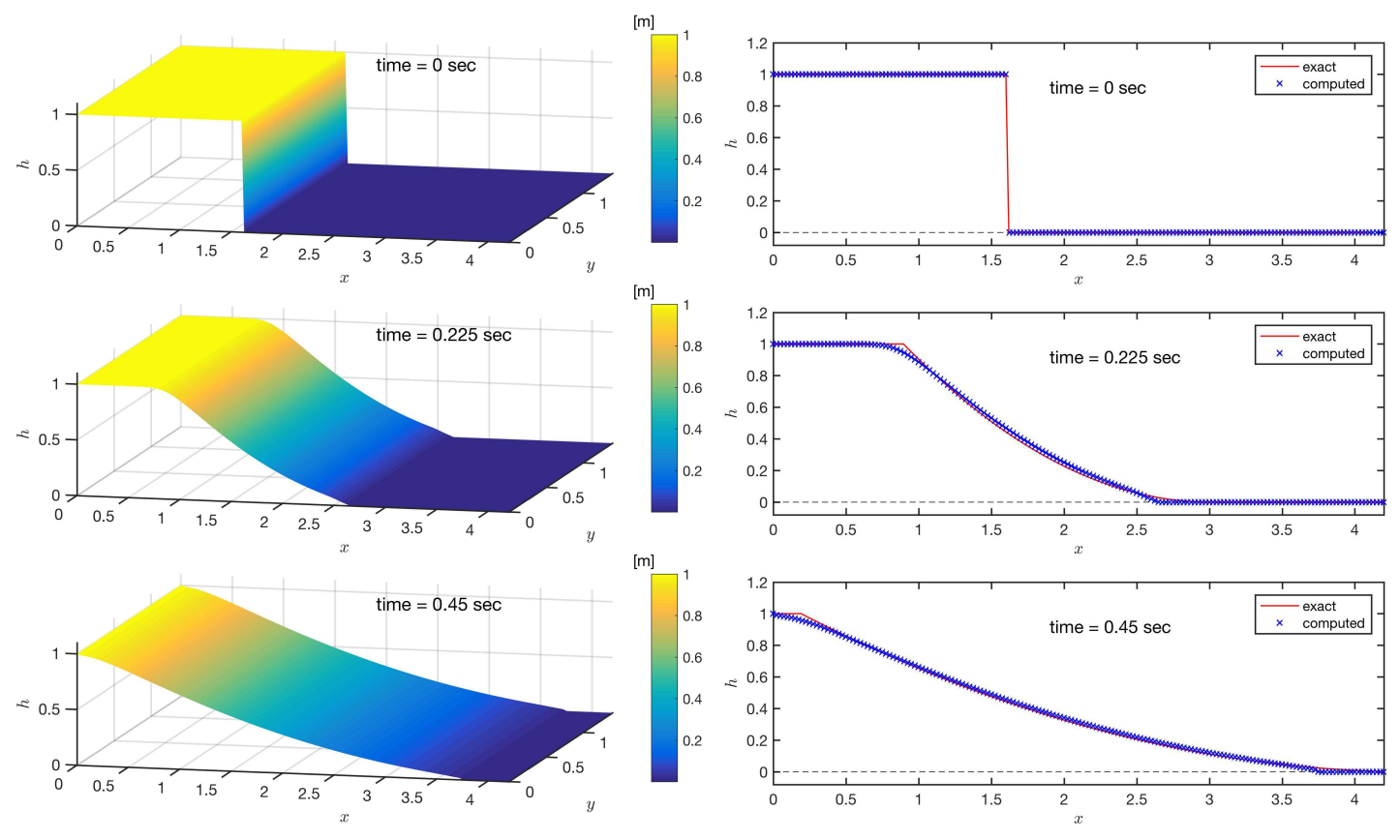

Figure 5: The depth evolution in the dry land case - Ritter's solution.

\section{Dam break flow: Stoker's exact solution}

The initial condition for the Stoker's test is defined with

$$
h_{L}=1.0 \mathrm{~m} \quad \text { and } \quad h_{R}=0.1 \mathrm{~m} \text {. }
$$

Then, the exact solution is composed by a left-going rarefaction wave connecting the initial left depth $h_{L}$ to a constant non-vanishing depth $h_{M}$ by a concave parabola, and at the right end of the constant depth $h_{M}$ a right-going shock that relates $h_{M}$ and $h_{R}$. The parabola ranges from $x_{A}(t)=x_{0}-t \sqrt{g h_{L}}$ to $x_{B}(t)=x_{0}+t\left(u_{M}-\sqrt{g h_{M}}\right)$, and the zone of constant depth lies in $\left[x_{B}(t), x_{C}(t)\right]$. Position $x_{C}(t)$ denotes the location of the shock wave moving with a constant velocity $u_{\text {shock}}$. The derivation of this Stoker's solution is tedious, so we just list the results and refer the readers to e.g. [56] for details. The velocity $u_{\text {shock }}$ is the solution of

$$
4\left(4 \Pi_{A}^{-1 / 2}+1\right) u_{\text {shock }}^{2}-8 \sqrt{g h_{L}} u_{\text {shock }}-g h_{R} \Pi_{A}=0
$$

with $\Pi_{A}=1+\left(1+8 u_{\text {shock }}^{2} /\left(g h_{R}\right)\right)^{1 / 2}$. Once the shock velocity $u_{\text {shock }}$ is determined, the thickness of the bore $h_{M}$ and the value of $u_{M}$ can be determined by the relations $g h_{M}=$ $4 u_{\text {shock }}^{2} / \Pi_{A}$, and $u_{M}=\left(1-h_{R} / h_{M}\right) u_{\text {shock, }}$ respectively (see e.g. [56]). Hence, for $t>0$, the 

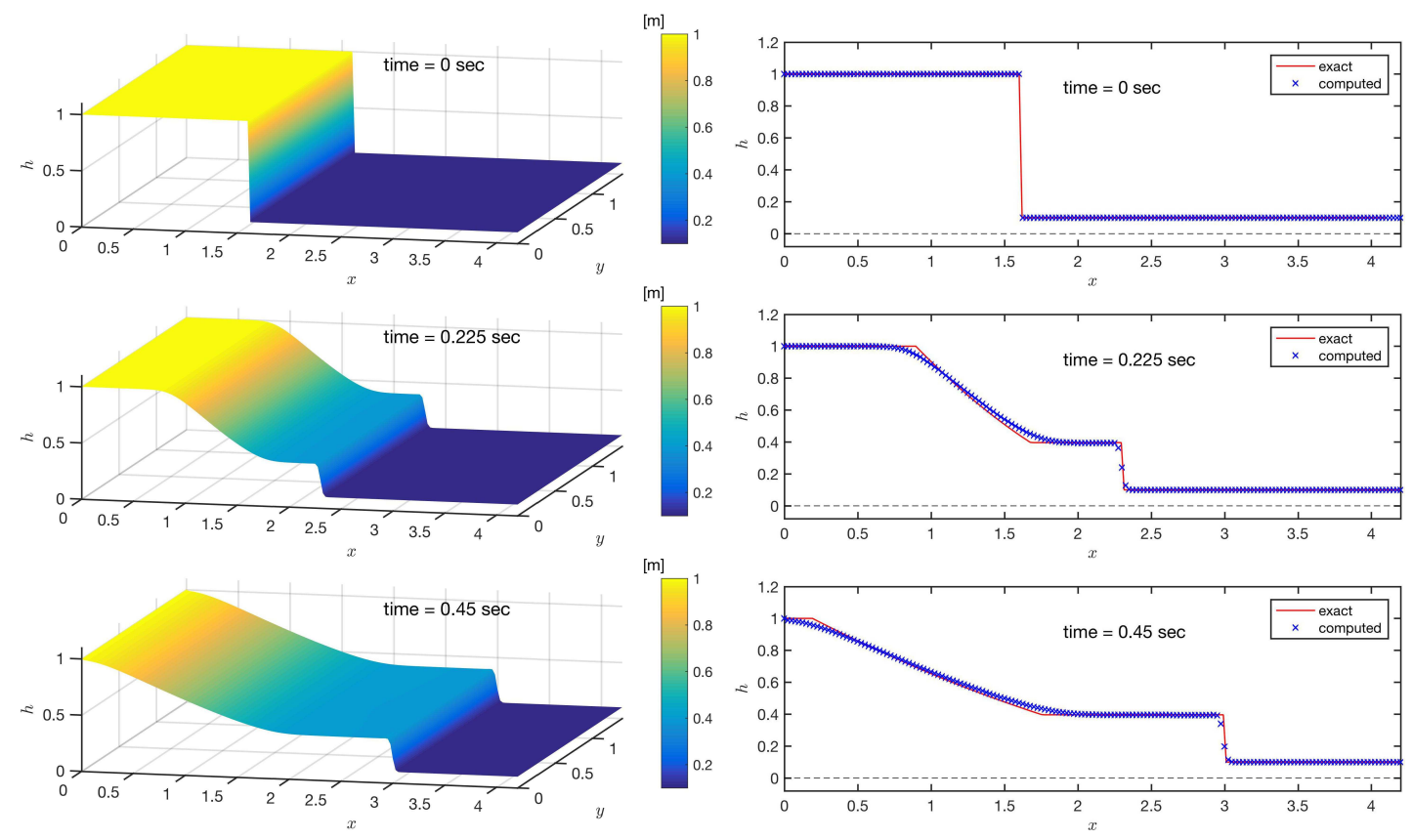

Figure 6: The depth evolution in the wet land case - Stoker's solution.

depth distribution reads

$$
h(x, y, t)= \begin{cases}h_{L} & \text { for } x \leq x_{A}(t), \\ \frac{4}{9 g}\left(\sqrt{g h_{L}}-\frac{x-x_{0}}{2 t}\right)^{2} & \text { for } x_{A}(t)<x<x_{B}(t), \\ h_{M} & \text { for } x_{B}(t)<x<x_{C}(t), \\ h_{R} & \text { for } x_{C}(t) \leq x,\end{cases}
$$

and

$$
u(x, y, t)= \begin{cases}0.0 & \text { for } x \leq x_{A}(t), \\ \frac{2}{3}\left(\sqrt{g h_{L}}+\frac{x-x_{0}}{t}\right) & \text { for } x_{A}(t)<x<x_{B}(t), \\ 2\left(\sqrt{g h_{L}}-\sqrt{g h_{M}}\right) & \text { for } x_{B}(t)<x<x_{C}(t), \\ 0.0 & \text { for } x_{C}(t) \leq x,\end{cases}
$$

represents the corresponding velocity distribution.

Now we compare the computed results and the above analytical solution both for depth and velocity, as shown in Figs. 6 and 7, respectively. Similar to the illustration in Fig. 5, the red lines represent the analytical solutions and the computed ones are denoted by blue cross-markers " $\times$ ".

For both dam-break problems considered in this section, the numerical solutions fit well with the exact solutions. Mesh convergence has been performed and optimal con- 

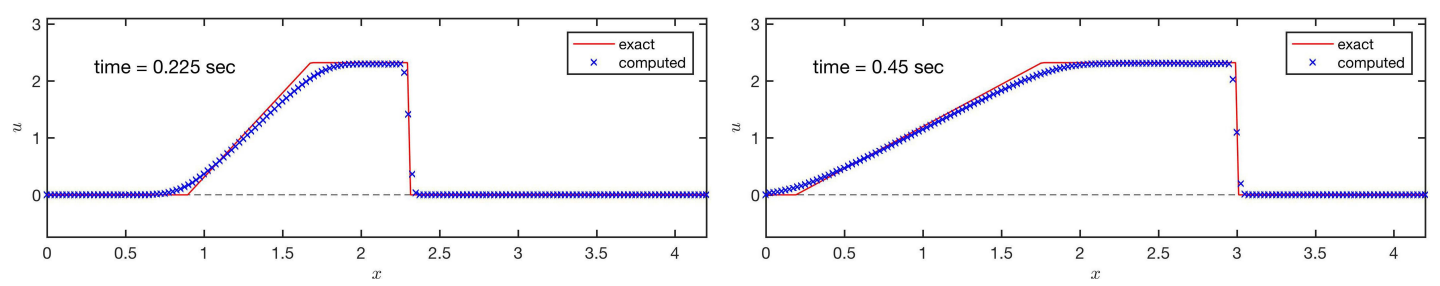

Figure 7: The velocity distributions in the wet land case - Stoker's solution.

vergence of first order is achieved for these non-smooth exact solutions, even if no special treatment is performed at the wet-dry moving boundary. According to the numerical analysis theory, the first order we have obtained is the optimal convergence expected in this context where analytical discontinuous solutions are available. On the strength of this result, we can therefore safely use this strategy for more complex applications.

\subsection{Quasi-1D flows on an inclined flat plane: Parabolic similarity solution}

One particular similarity solution in the one-dimensional case was proposed in [58] and has been generalized in [59], where a finite mass of granular material slides along a flat plane keeping the depth distribution in a parabolic shape along the down-slope direction. In this example, a flat plane with an inclination angle $\theta_{c}=35^{\circ}$ is considered, on which a local coordinate system $O_{\xi \eta \zeta}$ is assigned with the $\xi$-axis pointing towards the down-slope direction, the $\eta$-axis towards the cross-slope direction and the $\zeta$-axis pointing upwards normal to the plane. With respect to the multi-mesh-scale approximation proposed in Section 2, this inclined flat plane serves as the topography, i.e., $z=b(x, y)$, over the horizontal $x y$-plane:

$$
b(x, y)=\left(x_{R}-x\right) \tan \theta_{c} \quad \text { with } \quad(x, y) \in \Omega .
$$

Here, a rectangular domain $\Omega=[0,4.2 \mathrm{~m}] \times[0,1.4 \mathrm{~m}]$ and a "structured" triangular mesh, of $169 * 57$ vertices and 11816 triangular elements, are employed.

In the coordinate system $O_{\tilde{\xi} \eta \zeta}$, the initial depth is uniform in the $\eta$-direction and exhibits a parabolic shape along the down-slope $\xi$-direction, namely

$$
h(\xi, \eta, t=0)= \begin{cases}h_{0}\left(1.0-\left(\frac{\xi-0.46}{0.24}\right)^{2}\right) & \text { for }|\xi-0.46| \leq 0.24 \\ 0.0 & \text { elsewhere }\end{cases}
$$

where the flow thickness $h$ is measured normal to the plane and $h_{0}=0.1 \mathrm{~m}$ is the maximum depth located at $\xi=0.46$. For the initial velocity $\left(u_{\xi}, v_{\eta}\right)$ tangential to the flat plane, a linear distribution of velocity in the down-slope direction is assigned to the moving 

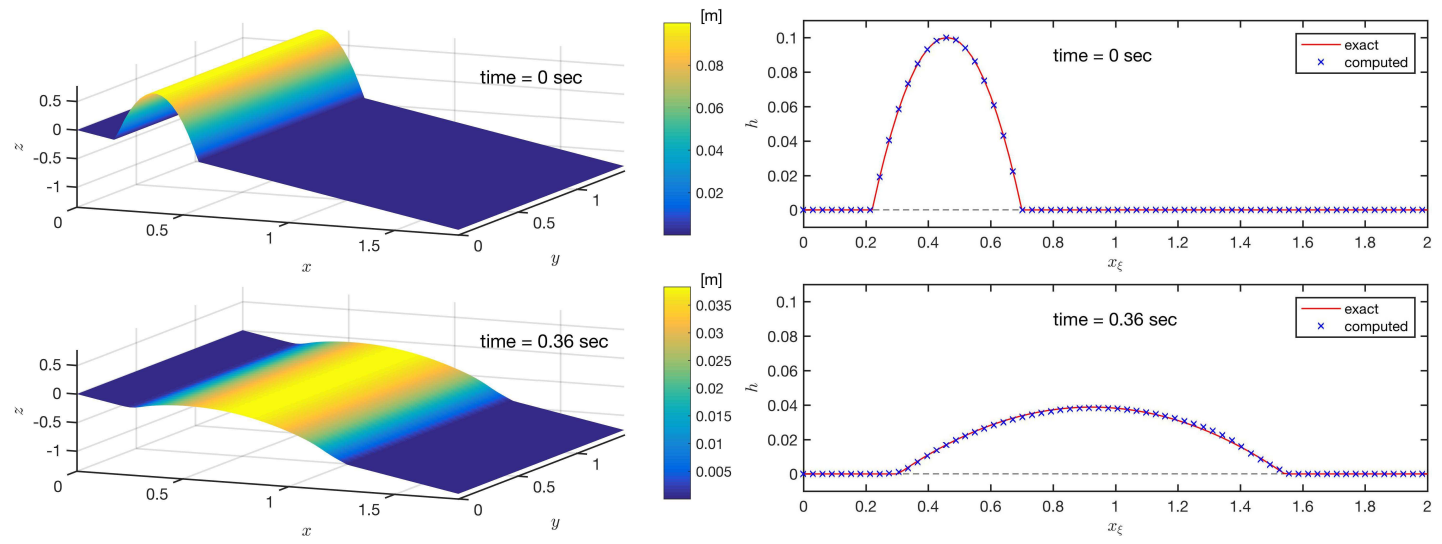

Figure 8: The depth evolution in the case of the similarity solution.

body, whilst no speed is given in the lateral direction. That is,

$$
\begin{aligned}
& u_{\xi}(\xi, \eta, t=0)= \begin{cases}u_{\xi c}\left(1.0+0.4\left(\frac{\xi-0.46}{0.24}\right)\right) & \text { for }|\xi-0.46| \leq 0.24, \\
0.0 & \text { elsewhere, }\end{cases} \\
& v_{\eta}(\xi, \eta, t=0)=0.0,
\end{aligned}
$$

where $u_{\xi c}=0.8 \mathrm{~m} / \mathrm{s}$ is the initial velocity of the center of the parabolic moving mass.

In the computation, the so-called earth-pressure coefficient introduced in [59] is set to be unity, so that the center of the moving mass slides with velocity

$$
u_{\tilde{\xi}}^{\text {center }}(t)=u_{\xi_{c}}+\int_{0}^{t} g\left\{\sin \theta_{c}-\mu \cos \theta_{c}\right\} d t^{\prime}
$$

in which $\mu$ is the Coulomb friction coefficient as introduced in (2.32). As time increases, the moving body extends with respect to the center symmetrically, keeping the parabolic shape and a linear distribution for the velocity along the down-slope direction ( $\xi$-direction). Since the total mass should be conserved, the moving body becomes flatter and flatter as it extends. Fig. 8 illustrates the depth distributions at $t=0.0$ and $0.36 \mathrm{~s}$, respectively. The corresponding section views along the central line $(y=0.7)$ are shown in the right panels, in which the red lines represent the analytical solutions and the blue " $\times$ " markers denote the numerical predictions.

\section{Application to back-calculation of a real event}

The proposed method is further applied to back-calculation of a real event, the Hsiaolin landslide, which took place during the typhoon Morakot in August 2009. Within 72 hours, an abnormal heavy rainfall of more than 2,000 $\mathrm{mm}$ accumulated precipitation 

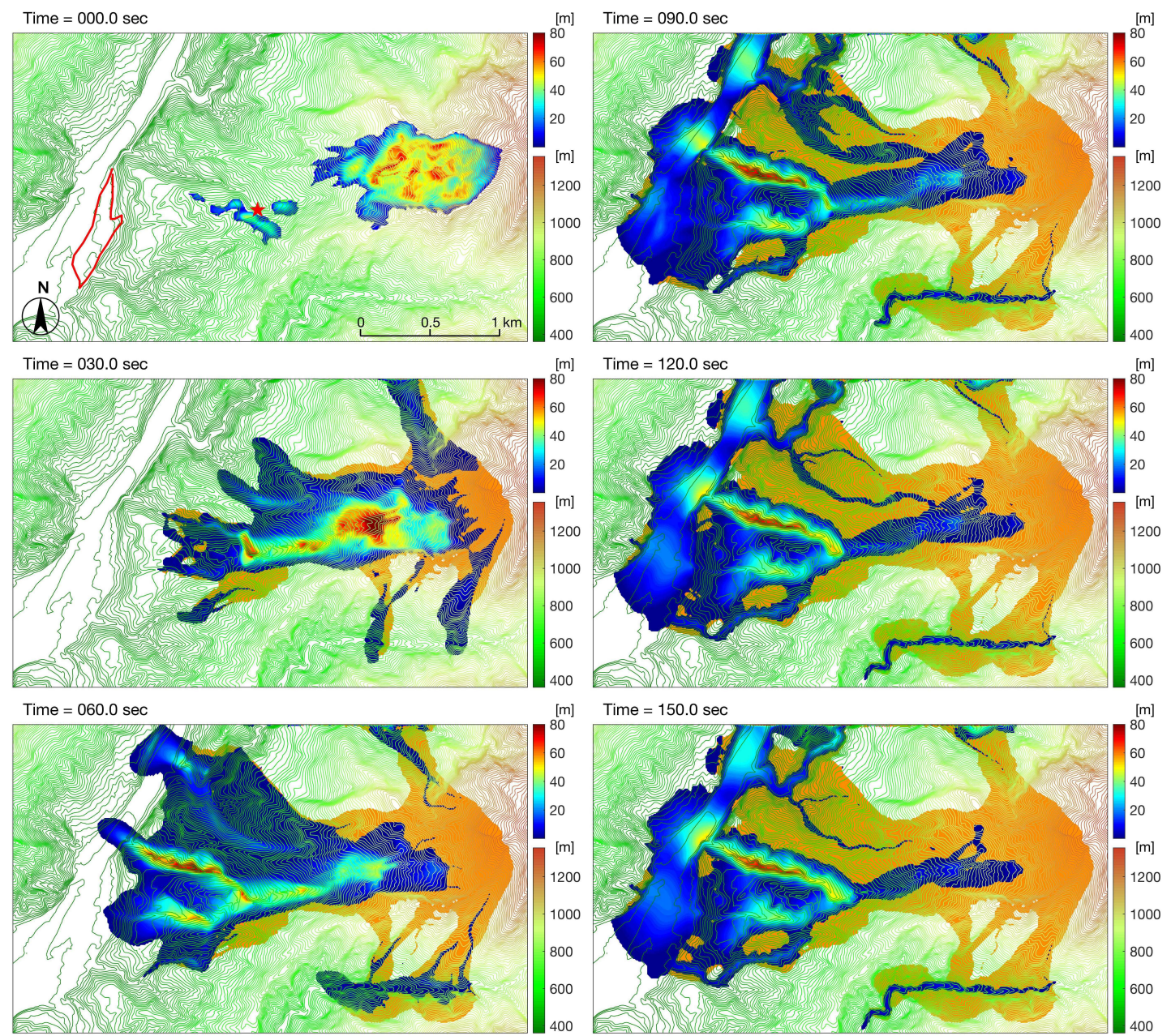

Figure 9: Evolution of the flow thickness, where the area covered by the dark brown color indicates the scoured area (source area and flow paths) at the corresponding time level. The topographic elevation is given by the colored contours with respect to the lower color bar, and the flow thickness is given with respect to the upper color bar. The Hsiaolin village is marked by the red line and the 590 Height is indicated by the red petagram.

triggered a large-scale landslide and the subsequent debris flow attached and buried the Hsiaolin village in southern Taiwan. It is reported that there were more than 470 victims in this event (see e.g., $[35,60])$. The post-disaster field investigation indicates that the major landslide body had an extent of $57 \times 10^{4} \mathrm{~m}^{2}$ with a mean depth of $42 \pm 3 \mathrm{~m}$ (see [61]).

In our application, the computational domain covers an area of 3,700 $\mathrm{m} \times 2,210 \mathrm{~m}$ with $371 \times 222$ vertices and 163,540 triangle elements in total. The applied angle of basal friction is set at $12.5^{\circ}$, which lies in the range given by the empirical regression formula for the friction coefficient [62]. In Figs. 9 and 10, the topography is depicted by the colored contours, where the elevation ranges from 353 to $1,363 \mathrm{~m}$, as indicated in the lower color bar. Fig. 9 shows the evolution of the flow thickness at sequential time levels, where 

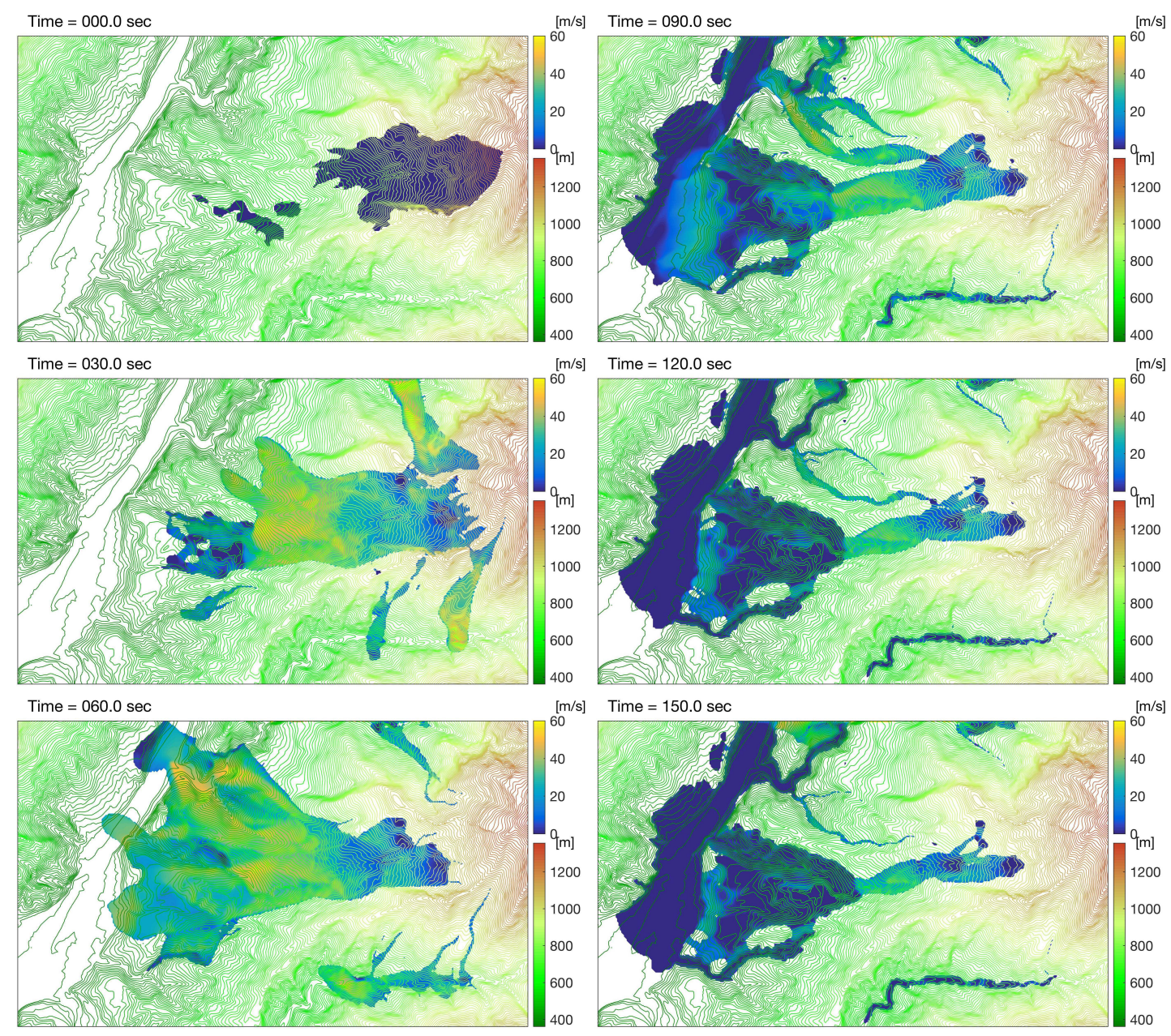

Figure 10: Velocity distributions of the moving mass at sequential time levels, as shown in Fig. 9. The topographic elevation is given by the colored contours with respect to the lower color bar, and the flow speed is given with respect to the upper color bar.

the top-right color bar indicates the corresponding thickness. The area covered by the dark brown color represents the scoured area (source area and flow paths) from initiation $t=0$ to the time level shown at the top of each panel. The mass is released from rest, as employed in [35] and shown in the panel at $t=0 \mathrm{~s}$, where the thick red line marks the area of Hsiaolin village. Approximately at the center of the figure stands a ridge, the 590 Height marked by a red star marker, which is capable of protecting the village when the amount of the moving mass is not too large. At $t=30 \mathrm{~s}$, the moving mass accumulates in front of the 590 Height and exhibits high flow depth. Due to the abnormally huge volume, part of the moving mass flows over the hill and towards the village (see the panel at $t=60 \mathrm{~s}$ ). One can recognize that there are approximately three branches: the northern branch, the middle main stream and the southern one. At this time level, the 

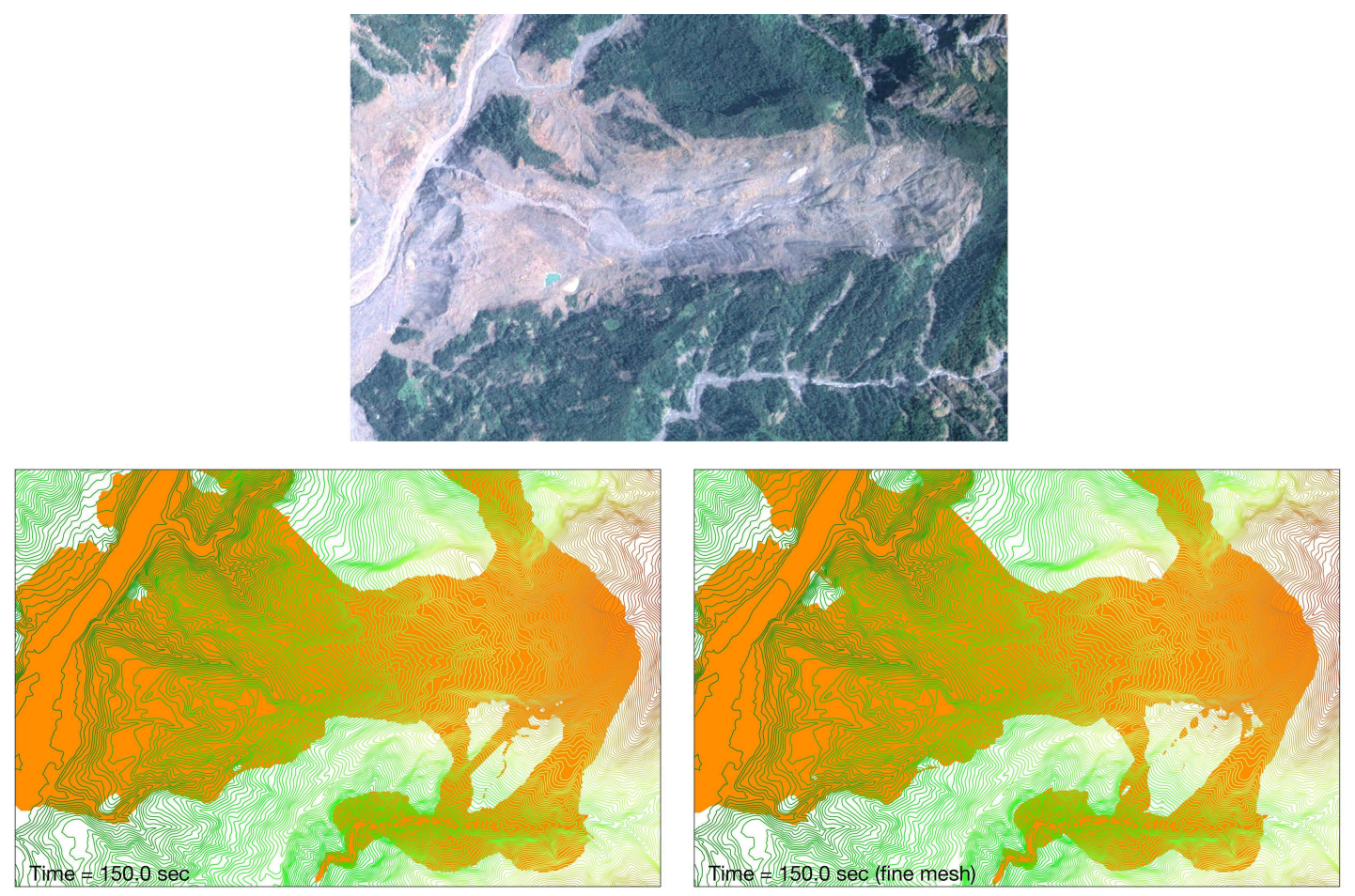

Figure 11: Top: the satellite image taken approximately 5 months after the event. Bottom: the computed scoured area (brown shade area) on a coarse mesh (left) and on a finer mesh (right).

debris just touches the village, while the northern and middle ones have arrived at the river channel. At $t=90 \mathrm{~s}$, the material slows down and begins to deposit, while the main stream collects most of the material and only a small part goes through the village. After $t=120 \mathrm{~s}$, the majority of the material has come to rest, and as a consequence, the distribution of flow thickness does not significantly change from $t=120 \mathrm{~s}$ to $t=150 \mathrm{~s}$.

The corresponding velocity (speed) distributions with respect to Fig. 9 are illustrated in Fig. 10, where the flow speed is color-coded according to the upper color bar. At $t=0$ $\mathrm{s}$, the moving mass is released from rest. The 590 Height retards the moving debris, so that significant velocity reduction can be found behind the ridge, see the panel for $t=30$ $\mathrm{s}$. The flow front begins to deposit once it crosses the river channel and touches the river bank $(t=60 \mathrm{~s})$, while the highest speed reaches $60 \mathrm{~m} / \mathrm{s}$. From $t=90 \mathrm{~s}$ to $t=150 \mathrm{~s}$, the speed generally corresponds to the local slope, and the material at the downstream area comes nearly to rest.

The top panel of Fig. 11 is a satellite image taken ca. 5 months after the event, where the bare area approximately depicts the flow paths, including the source scar and deposit area. The flow-impacted areas (source scar, flow paths and deposit area) in the computation are illustrated by the brown shades in the bottom panels of Fig. 11. The left panel shows the results as given in Fig. 9 and the right panel show the results computed with 

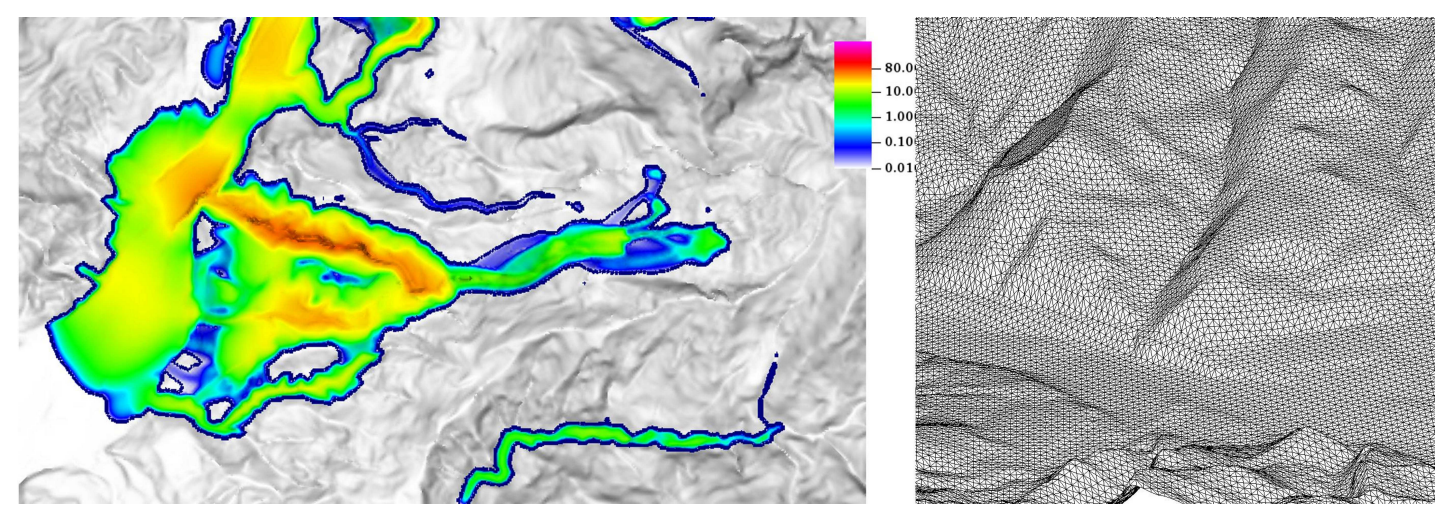

Figure 12: The numerical results at time $t=150 \mathrm{~s}$ on a structured triangular mesh. Left: the flow thickness; right: zoom on the mesh.

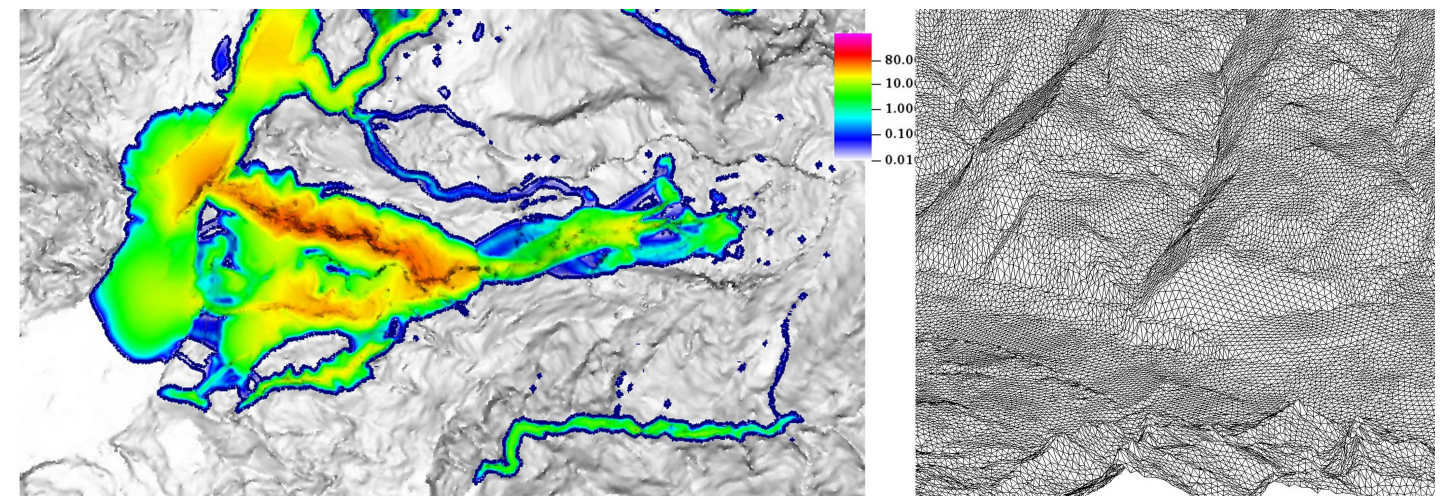

Figure 13: Numerical results at time $t=150 \mathrm{~s}$ on an unstructured triangular mesh (obtained through interpolation). Left: the flow thickness; right: zoom on the mesh.

a finer mesh (double the amount of vertices in each direction), where no significant discrepancy (concerning the brown shade area) between the bottom panels is recognized. It reveals that the solution converges with mesh-refinement. Comparing the flow impact area (from $t=0 \mathrm{~s}$ to $t=150 \mathrm{~s}$ with $6 \mathrm{~s}$ increment and indicated by the brown shade) with the satellite image (the bare area), the computed results cover a slightly larger area than that in the satellite image. Especially at the northern and southern flanks of the source area, more mass slides sideways and over the ridges. This overflow is suspected to be caused by the initial condition, in which the whole mass is suddenly released.

The results shown in Figs. 9-11 are computed with a structured triangular mesh. The present approach is well applicable to an unstructured mesh. For ease of comparison, Fig. 12 shows the flow thickness (left panel) at time $t=150 \mathrm{~s}$ with a structured triangular mesh, where the zoomed mesh pattern is shown in the right panel. The distribution of the flow thickness at $t=150 \mathrm{~s}$ and the employed unstructured mesh are illustrated in Fig. 13, where the topography and initial depth are obtained by interpolation with respect to the data used in the computations for Figs. 9 and 10. In both Figs. 12 and 13, the margin 
indicates the location of the flow thickness of $1 \mathrm{~cm}$. Although discrepancy of the margin locations for the flow thickness can be identified, the distributions of the material are rather similar. In fact, even if the interpolated topography is globally similar to the initial one, there are locally some differences that can explain the shifting in the computed wave front. However, the global shapes and the distributions of the flow thickness are similar.

\section{Concluding remarks}

In the present study we have proposed a multi-mesh-scale approach for describing the dynamic behaviors of shallow flows over complex topographies. Since the topographic surface is of non-trivial geometry, it is virtually impossible to define a non-singular mapping that fits the basal surface everywhere. Moreover, the derivation of a global depth average is questionable. It is for this reason that we have proposed a numerical strategy that can be viewed as a 3D finite volume approximation of the free surface incompressible Navier-Stokes equations. Locally, there is a single volume in the normal direction to the basal surface. This dynamic 3D volume is defined by a surface cell fitting with the basal topography and an averaged depth. The cell surface is obtained in the vertex-centered framework on unstructured meshes of flat triangles. The depth of the 3D volume is an unknown of the problem. Therefore, depth average is locally found in the numerical approximation. The final numerical scheme is based on computations of fluxes found using approximated solutions of Riemann problems.

For the simple basal geometry, we have recovered the solution of the classical discretization of the shallow water equations with optimal rate of convergence to the exact solution. The application to the large-scale event, the Hsiaolin landslide, shows that the numerical computation reasonably retraces the landslide paths and deposit, where sound agreements can be identified with respect to the satellite picture and in-situ measurement. As well, no expensive resources are requested for the computation. For the computation of the Hsiaolin landslide event $(371 \times 222$ vertices and 163,540 triangle elements in total) with a single processor (core), the run time ranges from 0.8 hours (processor $3.60 \mathrm{GHz}$ Intel Core i-7-7700, 16 GB memory, Linux OS) to ca. 2 hours (processor $2.5 \mathrm{GHz}$ Intel Core i7, 16 GB memory, Mac-Book Pro). Hence, the proposed multi-mesh-scale approximation has a high potential for engineering applications as it provides more flexibility in the representation of the topography. In this context, it is easier and more useful to develop local refinement based on some specific criteria such as curvature or geological properties.

Although the results have shown the general applicability of the present approach, it should be noted that it follows the depth-averaged concept, so that some important effects or phenomena are neglected or still missing. For example, a shallow curvature with respect to the flow thickness and the topography is required. In addition, the nonuniform distribution of the velocity along the flow thickness is not taken into account yet. In experiments of geophysical mass flows, either for dry granular flows or for saturated 
grain-fluid debris flows where the impact of the viscosity is significant, the velocity profiles are generally non-uniform $[63,64]$. This non-uniformity introduces the correction (shape) factor $(>1)$ induced by the advective terms in the momentum equations, while the non-unity value of the correction factor will change the characteristic structure of the equation system (e.g., [65]), so that additional treatment is needed. Another issue is the non-hydrostatic pressure, which could be induced by the non-uniform velocity profile $[48,49,63]$, grain-size-distribution [66], or the so-called enhanced gravity (excess pore fluid pressure induced by granular dilatancy) $[3,37,66-68]$. These neglected phenomena might explain the employment of a small angle of basal friction in our back-calculation for the Hsiaolin event. Two-phase models $[8,10,11,27,36,39]$ or the more advanced multiphase models [12], in which the multi-component Coulomb-viscoplastic mixture consisting of solid, fine-solid and fluid is considered that takes into account the multi-phase rheology, enhanced generalized drag and virtual mass forces, and Non-Newtonian viscous effects, might be the plausible solution, but more parameters would be needed in the model. As well, the erosion and deposition $[2,4,6,30,33,34]$ should be integrated into the numerical model for a more realistic scenario, where the determination of the evolution of the mesh system would play a crucial role. More efforts are requested on these tasks and we will report on the future results in due time.

\section{Acknowledgments}

We are grateful to the Ecological Soil and Water Conservation Research Center at National Cheng Kung University for the satellite image. Part of the results were conducted during the visit of J. Vides and B. Nkonga to the Department of Hydraulics and Ocean Engineering, NCKU, Taiwan, and the visit of Y.C. Tai and C.Y. Kuo to the University of Nice Côte d'Azur (UCA) in the framework of the Inria-MOST Associate team program AMoSS and funding MOST-105-2911-I-006-502. B. Nkonga and J. Vides acknowledge the warm hospitality of the NCTS at NTU Taipei. Y.C. Tai was partially supported by MOST-106-2221-E-006-056.

\section{Appendix}

\section{A.1 Surface integrals}

In order to derive the finite volume scheme, we need to be able to compute integrals of the form

$$
\int_{\mathcal{V}_{i}^{e}} \nabla \cdot \mathbf{U} d \widetilde{\boldsymbol{x}}=\int_{\partial \mathcal{V}_{i}^{e}} \mathbf{U} \cdot \boldsymbol{n} d S_{\widetilde{\boldsymbol{x}}} \text { and } \int_{\mathcal{V}_{i}^{e}} \nabla \cdot \underline{\boldsymbol{T}} d \widetilde{\boldsymbol{x}}=\int_{\partial \mathcal{V}_{i}^{e}} \underline{\boldsymbol{T}} \boldsymbol{n} d S_{\widetilde{\boldsymbol{x}}}
$$

where $\mathcal{V}_{i}^{e}$ is the considered element's volume with its boundary $\partial \mathcal{V}_{i}^{e}$ and $\underline{\mathbf{T}}=\mathbf{U} \otimes \mathbf{U}+\underline{\mathbf{P}}$. Let us recall that $\widetilde{\boldsymbol{x}}$ are the Cartesian coordinates, $\boldsymbol{n}$ is the outward unit normal vector in this system of coordinates and $d S_{\tilde{x}}$ is the surface increment. 
In this context, it is more convenient to use a local surface coordinate in the evaluation of the fluxes (A.1). Thus, the transformation (2.2) will be used, which we rewrite here for convenience:

$$
\widetilde{\boldsymbol{x}}(\widetilde{\boldsymbol{\xi}})=\boldsymbol{\xi}_{\tilde{\boldsymbol{g}}}+\eta \boldsymbol{g}_{\eta}+\zeta \boldsymbol{g}_{\zeta}
$$

where $\boldsymbol{g}_{\tilde{\zeta}}, \boldsymbol{g}_{\eta}$ and $\boldsymbol{g}_{\zeta}$ are the basis vectors of the local coordinate and $\widetilde{\boldsymbol{\xi}}=(\xi, \eta, \zeta)^{\mathrm{T}}$. The stress tensor then reads

$$
\begin{aligned}
\underline{\boldsymbol{T}}= & T_{\xi \xi} \boldsymbol{g}^{\xi} \otimes \boldsymbol{g}_{\xi}+T_{\xi \eta} \boldsymbol{g}^{\xi} \otimes \boldsymbol{g}_{\eta}+T_{\xi \zeta} \boldsymbol{g}^{\xi} \otimes \boldsymbol{g}_{\zeta} \\
& +T_{\eta \xi} \boldsymbol{g}^{\eta} \otimes \boldsymbol{g}_{\zeta}+T_{\eta \eta} \boldsymbol{g}^{\eta} \otimes \boldsymbol{g}_{\eta}+T_{\eta \zeta} \boldsymbol{g}^{\eta} \otimes \boldsymbol{g}_{\zeta} \\
& +T_{\zeta \zeta} \boldsymbol{g}^{\zeta} \otimes \boldsymbol{g}_{\xi}+T_{\zeta \eta} \boldsymbol{g}^{\zeta} \otimes \boldsymbol{g}_{\eta}+T_{\zeta \zeta} \boldsymbol{g}^{\zeta} \otimes \boldsymbol{g}_{\zeta} .
\end{aligned}
$$

We also urge the reader to recall that the Jacobian of the transformation is

$$
\mathrm{J}=\left(\boldsymbol{g}_{\xi} \times \boldsymbol{g}_{\eta}\right) \cdot \boldsymbol{g}_{\zeta}
$$

as was introduced in (2.3). Let us now compute the integrals (A.1) on three different types of surfaces: basal, flow and side surfaces.

\section{On basal surfaces}

In this case, the surface is only parametrized by $\xi$ and $\eta$, and $\widetilde{\boldsymbol{x}}(\widetilde{\boldsymbol{\xi}})=\xi \boldsymbol{g}_{\xi}+\eta \boldsymbol{g}_{\eta}$. First, for the continuity equation, we get

$$
\begin{aligned}
\int_{\mathcal{C}_{i}^{e}} \mathbf{U} \cdot \boldsymbol{n} d S_{\tilde{\boldsymbol{x}}} & =-\int_{\tilde{\xi}} \int_{\eta} \mathbf{U} \cdot\left(\frac{\partial \widetilde{\boldsymbol{x}}}{\partial \xi} d \xi \times \frac{\partial \widetilde{\boldsymbol{x}}}{\partial \eta} d \eta\right) \\
& =-\int_{\tilde{\xi}} \int_{\eta} \mathbf{U} \cdot\left(\boldsymbol{g}_{\xi} d \xi \times \boldsymbol{g}_{\eta} d \eta\right) \\
& =-\int_{\xi} \int_{\eta} u^{\zeta} \boldsymbol{g}_{\zeta} \cdot\left(\boldsymbol{g}_{\xi} d \xi \times \boldsymbol{g}_{\eta} d \eta\right) \\
& =-\int_{\xi} \int_{\eta} \mathrm{J} u^{\zeta} d \xi d \eta
\end{aligned}
$$

and

$$
\begin{aligned}
a_{i}^{e} & =\int_{\xi} \int_{\eta} \frac{\boldsymbol{g}_{\xi} \times \boldsymbol{g}_{\eta}}{\left\|\boldsymbol{g}_{\xi} \times \boldsymbol{g}_{\eta}\right\|} \cdot\left(\boldsymbol{g}_{\xi} d \xi \times \boldsymbol{g}_{\eta} d \eta\right) \\
& =\int_{\xi} \int_{\eta} \boldsymbol{g}_{\zeta} \cdot\left(\boldsymbol{g}_{\xi} d \xi \times \boldsymbol{g}_{\eta} d \eta\right) \\
& =\int_{\xi} \int_{\eta} \mathrm{J} d \xi d \eta
\end{aligned}
$$


where $a_{i}^{e}=\mathrm{J} / 6$ for the triangular shape of the local element. Then, for the momentum equation, the associated integral is

$$
\begin{aligned}
\int_{\mathcal{C}_{i}^{e}} \underline{\mathbf{T}} n d S_{\tilde{x}} & =-\int_{\tilde{\zeta}} \int_{\eta} \underline{\mathbf{T}}\left(\boldsymbol{g}_{\xi} d \xi \times \boldsymbol{g}_{\eta} d \eta\right)=-\int_{\tilde{\xi}} \int_{\eta} \underline{\mathbf{T}}\left(\boldsymbol{g}_{\xi} \times \boldsymbol{g}_{\eta}\right) d \xi d \eta \\
& =-\int_{\xi} \int_{\eta} \mathbf{J} \underline{\mathbf{T}} \boldsymbol{g}^{\zeta} d \xi d \eta .
\end{aligned}
$$

\section{On flow surfaces}

Here, the surface is parametrized by $\xi$ and $\eta: \widetilde{\boldsymbol{x}}(\widetilde{\boldsymbol{\xi}})=\xi \boldsymbol{g}_{\xi}+\eta \boldsymbol{g}_{\eta}+h(t, \xi, \eta) \boldsymbol{g}_{\zeta}$. Thus, the surface integral associated to the continuity equation becomes

$$
\begin{aligned}
\int_{\mathcal{C}_{i}^{e}} \mathbf{U} \cdot \boldsymbol{n} d S_{\widetilde{\boldsymbol{x}}} & =\int_{\xi} \int_{\eta} \mathbf{U} \cdot\left(\frac{\partial \widetilde{\boldsymbol{x}}}{\partial \xi} d \xi \times \frac{\partial \widetilde{\boldsymbol{x}}}{\partial \eta} d \eta\right)=\int_{\xi} \int_{\eta} \mathbf{U} \cdot\left(\left(\boldsymbol{g}_{\xi}+\partial_{\xi} h \boldsymbol{g}_{\zeta}\right) d \xi \times\left(\boldsymbol{g}_{\eta}+\partial_{\eta} h \boldsymbol{g}_{\zeta}\right) d \eta\right) \\
& =\int_{\xi} \int_{\eta} \mathbf{U} \cdot\left(\boldsymbol{g}_{\xi} \times \boldsymbol{g}_{\eta}+\partial_{\eta} h \boldsymbol{g}_{\xi} \times \boldsymbol{g}_{\zeta}+\partial_{\xi} h \boldsymbol{g}_{\zeta} \times \boldsymbol{g}_{\eta}\right) d \xi d \eta \\
& =\int_{\xi} \int_{\eta} \mathbf{J} \cdot\left(\boldsymbol{g}^{\zeta}-\partial_{\eta} h \boldsymbol{g}^{\eta}-\partial_{\xi} h \boldsymbol{g}^{\tau}\right) d \xi d \eta \\
& =\int_{\xi} \int_{\eta}\left(u^{\zeta}-\partial_{\xi} h u^{\xi}-\partial_{\eta} h u^{\eta}\right) \mathrm{J} d \xi d \eta,
\end{aligned}
$$

where the relations $\boldsymbol{g}^{\xi}=\frac{1}{\mathrm{~J}}\left(\boldsymbol{g}_{\eta} \times \boldsymbol{g}_{\zeta}\right), \boldsymbol{g}^{\eta}=\frac{1}{\mathrm{~J}}\left(\boldsymbol{g}_{\zeta} \times \boldsymbol{g}_{\zeta}\right)$ and $\boldsymbol{g}^{\zeta}=\boldsymbol{g}_{\zeta}=\frac{1}{\mathrm{~J}}\left(\boldsymbol{g}_{\xi} \times \boldsymbol{g}_{\eta}\right)$ have been used. In addition, the stress term in the momentum equation yields

$$
\begin{aligned}
\int_{\mathcal{C}_{i}^{e}} \underline{\boldsymbol{T}} n d S_{\widetilde{\boldsymbol{x}}} & =\int_{\xi} \int_{\eta} \underline{\boldsymbol{T}}\left(\boldsymbol{g}_{\xi} \times \boldsymbol{g}_{\eta}+\partial_{\eta} h \boldsymbol{g}_{\xi} \times \boldsymbol{g}_{\zeta}+\partial_{\xi} h \boldsymbol{g}_{\zeta} \times \boldsymbol{g}_{\eta}\right) d \xi d \eta \\
& =\int_{\xi} \int_{\eta} \underline{\mathbf{T}}\left(\boldsymbol{g}^{\zeta}-\partial_{\eta} h \boldsymbol{g}^{\eta}-\partial_{\xi} h \boldsymbol{g}^{\xi}\right) d \xi d \eta
\end{aligned}
$$

\section{On side surfaces}

In this last case, the surface is parametrized by $\ell$ and $\zeta$, such that

$$
\widetilde{\boldsymbol{x}}(\widetilde{\boldsymbol{\xi}})=\widetilde{\boldsymbol{x}}_{0}+\ell\left(\alpha_{\xi} \boldsymbol{g}_{\xi}+\alpha_{\eta} \boldsymbol{g}_{\eta}\right)+\zeta \boldsymbol{g}_{\zeta}
$$

with $\alpha_{\xi}$ and $\alpha_{\eta}$ being two constants. Then, the surface integral over $\partial \mathcal{V}_{i}^{e} \cap \partial \mathcal{V}_{m}^{e}$ is

$$
\begin{aligned}
\int_{\partial \mathcal{V}_{i}^{e} \cap \partial \mathcal{V}_{m}^{e}} \mathbf{U} \cdot \boldsymbol{n} d S_{\widetilde{\boldsymbol{x}}} & =\int_{\ell} \int_{\zeta} \mathbf{U} \cdot\left(\frac{\partial}{\partial \ell} \widetilde{\boldsymbol{x}} d \ell \times \frac{\partial}{\partial \zeta} \widetilde{\boldsymbol{x}} d \zeta\right)=\int_{\ell} \int_{\zeta} \mathbf{U} \cdot\left[\left(\alpha_{\zeta} \boldsymbol{g}_{\xi}+\alpha_{\eta} \boldsymbol{g}_{\eta}\right) \times \boldsymbol{g}_{\zeta}\right] d \ell d \zeta \\
& =\int_{\ell} \int_{\zeta} \mathbf{U} \cdot\left[\alpha_{\zeta}\left(\boldsymbol{g}_{\xi} \times \boldsymbol{g}_{\zeta}\right)+\alpha_{\eta}\left(\boldsymbol{g}_{\eta} \times \boldsymbol{g}_{\zeta}\right)\right] d \ell d \zeta \\
& =\int_{\ell} \int_{\zeta} \mathrm{JU} \cdot\left(-\alpha_{\xi} \boldsymbol{g}^{\eta}+\alpha_{\eta} \boldsymbol{g}^{\tau}\right) d \ell d \zeta \\
& =\int_{\ell} \int_{\zeta} \mathrm{J}\left(u^{\xi} \boldsymbol{g}_{\xi} \cdot \widetilde{\boldsymbol{n}}+u^{\eta} \boldsymbol{g}_{\eta} \cdot \widetilde{\boldsymbol{n}}\right) d \ell d \zeta,
\end{aligned}
$$


where the normal vector $\widetilde{\boldsymbol{n}}$ is given by

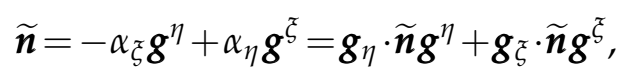

with $\alpha_{\xi}=-\boldsymbol{g}_{\eta} \cdot \widetilde{\boldsymbol{n}}$ and $\alpha_{\eta}=\boldsymbol{g}_{\xi} \cdot \widetilde{\boldsymbol{n}}$. Proceeding in the same way, we get, for any tensor $\underline{\boldsymbol{T}}$,

$$
\int_{\partial \mathcal{V}_{i}^{e} \cap \partial \mathcal{V}_{m}^{e}} \underline{\boldsymbol{T}} \boldsymbol{n} d S_{\widetilde{\boldsymbol{x}}}=\int_{\ell} \int_{\zeta} \mathrm{J} \underline{\boldsymbol{T}} \widetilde{\boldsymbol{n}} d \ell d \zeta=\int_{\ell} \int_{\zeta} \mathrm{J}\left(\boldsymbol{g}_{\zeta} \cdot \widetilde{\boldsymbol{n}} \underline{\mathbf{T}} \boldsymbol{g}^{\zeta}+\boldsymbol{g}_{\eta} \cdot \widetilde{\boldsymbol{n}} \underline{\mathbf{T}} \boldsymbol{g}^{\eta}\right) d \ell d \zeta .
$$

\section{A.2 Interface normal vectors on a triangular element}

In practice, we have plane surfaces for which the normal vectors are constant, and formulations with the covariant vectors are straightforward.

Let us consider a given element defined by the points $\overline{\widetilde{\boldsymbol{x}}}_{i}, \overline{\widetilde{\boldsymbol{x}}}_{j}$ and $\overline{\boldsymbol{x}}_{k}$, oriented as in Fig. 14. In order to obtain a finite volume approximation, we need to be able to define cell interface normal vectors. For this, on the considered triangular element, we first introduce the midpoints

$$
\overline{\overline{\boldsymbol{x}}}_{i j}=\frac{\overline{\boldsymbol{x}}_{i}+\overline{\widetilde{\boldsymbol{x}}}_{j}}{2}, \quad \overline{\overline{\boldsymbol{x}}}_{j k}=\frac{\overline{\widetilde{\boldsymbol{x}}}_{j}+\overline{\overline{\boldsymbol{x}}}_{k}}{2}, \quad \overline{\widetilde{\boldsymbol{x}}}_{k i}=\frac{\overline{\widetilde{\boldsymbol{x}}}_{k}+\overline{\widetilde{\boldsymbol{x}}}_{i}}{2},
$$

and the triangle's centroid

$$
\overline{\widetilde{\boldsymbol{x}}}_{g}=\frac{\overline{\boldsymbol{x}}_{i}+\overline{\overline{\boldsymbol{x}}}_{j}+\overline{\overline{\boldsymbol{x}}}_{k}}{3}
$$

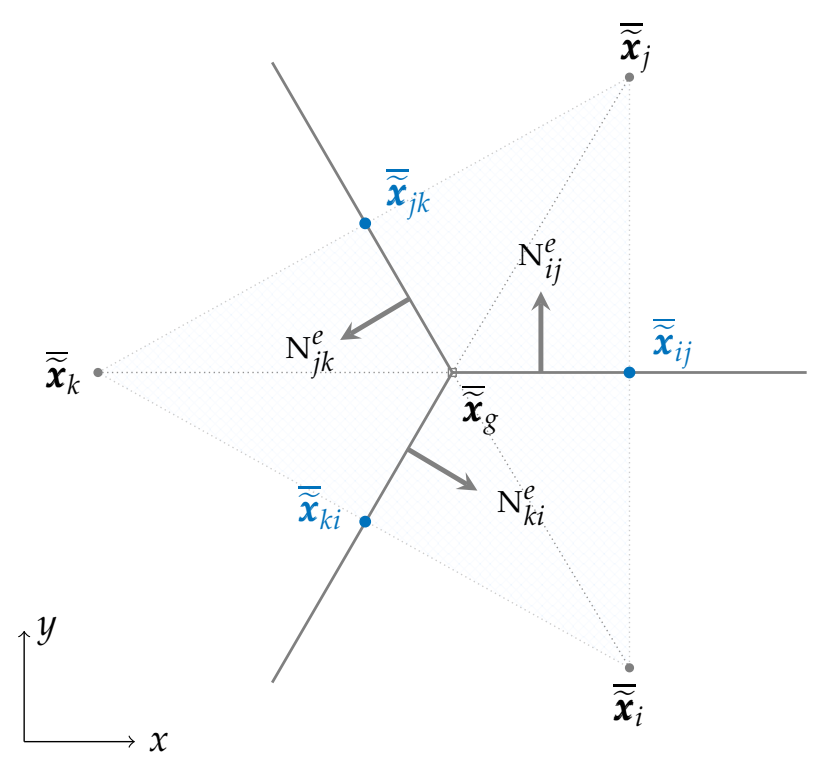

Figure 14: Integrated normal vectors on a triangular element. 
so that the integrated normal vectors are given by

$$
\left\{\begin{array}{c}
\mathrm{N}_{i j}^{e}=\int_{\ell_{i j}} \mathrm{~J} \widetilde{\boldsymbol{n}} d \ell=-\left(\overline{\widetilde{\boldsymbol{x}}}_{g}-\overline{\widetilde{\boldsymbol{x}}}_{i j}\right) \times \boldsymbol{g}_{\zeta}, \\
\mathrm{N}_{j k}^{e}=\int_{\ell_{j k}} \mathrm{~J} \widetilde{\boldsymbol{n}} d \ell=-\left(\overline{\boldsymbol{x}}_{g}-\overline{\boldsymbol{x}}_{j k}\right) \times \boldsymbol{g}_{\zeta}, \\
\mathrm{N}_{k i}^{e}=\int_{\ell_{k i}} \mathrm{~J} \widetilde{\boldsymbol{n}} d \ell=-\left(\overline{\boldsymbol{x}}_{g}-\overline{\boldsymbol{x}}_{k i}\right) \times \boldsymbol{g}_{\zeta},
\end{array}\right.
$$

where

$$
\begin{aligned}
& \overline{\widetilde{\boldsymbol{x}}}_{g}-\overline{\widetilde{\boldsymbol{x}}}_{i j}=\frac{1}{6}\left(-\overline{\widetilde{\boldsymbol{x}}}_{i}-\overline{\widetilde{\boldsymbol{x}}}_{j}+2 \overline{\widetilde{\boldsymbol{x}}}_{k}\right)=\frac{1}{6}\left(2\left(\overline{\widetilde{\boldsymbol{x}}}_{k}-\overline{\widetilde{\boldsymbol{x}}}_{i}\right)-\left(\overline{\widetilde{\boldsymbol{x}}}_{j}-\overline{\boldsymbol{\boldsymbol { x }}}_{i}\right)\right)=\frac{-\boldsymbol{g}_{\xi}+2 \boldsymbol{g}_{\eta}}{6}, \\
& \overline{\widetilde{\boldsymbol{x}}}_{g}-\overline{\widetilde{\boldsymbol{x}}}_{j k}=\frac{1}{6}\left(-\overline{\widetilde{\boldsymbol{x}}}_{j}-\overline{\widetilde{\boldsymbol{x}}}_{k}+2 \overline{\widetilde{\boldsymbol{x}}}_{i}\right)=\frac{1}{6}\left(-\left(\overline{\widetilde{\boldsymbol{x}}}_{k}-\overline{\widetilde{\boldsymbol{x}}}_{i}\right)-\left(\overline{\widetilde{\boldsymbol{x}}}_{j}-\overline{\widetilde{\boldsymbol{x}}}_{i}\right)\right)=\frac{-\boldsymbol{g}_{\xi}-\boldsymbol{g}_{\eta}}{6}, \\
& \overline{\widetilde{\boldsymbol{x}}}_{g}-\overline{\widetilde{\boldsymbol{x}}}_{k i}=\frac{1}{6}\left(-\overline{\widetilde{\boldsymbol{x}}}_{k}-\overline{\widetilde{\boldsymbol{x}}}_{i}+2 \overline{\widetilde{\boldsymbol{x}}}_{j}\right)=\frac{1}{6}\left(-\left(\overline{\boldsymbol{x}}_{k}-\overline{\widetilde{\boldsymbol{x}}}_{i}\right)+2\left(\overline{\widetilde{\boldsymbol{x}}}_{j}-\overline{\widetilde{\boldsymbol{x}}}_{i}\right)\right)=\frac{2 \boldsymbol{g}_{\xi}-\boldsymbol{g}_{\eta}}{6} .
\end{aligned}
$$

A relation that will be useful to derive simplified expressions for the above mentioned normal vectors is

$$
\begin{aligned}
\left(\alpha_{\eta} \boldsymbol{g}_{\eta}+\alpha_{\xi} \boldsymbol{g}_{\xi}\right) \times \boldsymbol{g}_{\zeta} & =\frac{1}{\mathrm{~J}}\left(\alpha_{\eta} \boldsymbol{g}_{\eta}+\alpha_{\xi} \boldsymbol{g}_{\xi}\right) \times\left(\boldsymbol{g}_{\xi} \times \boldsymbol{g}_{\eta}\right) \\
& =\frac{1}{\mathrm{~J}}\left(\alpha_{\xi} \boldsymbol{g}_{\xi} \cdot \boldsymbol{g}_{\eta}+\alpha_{\eta} \boldsymbol{g}_{\eta} \cdot \boldsymbol{g}_{\eta}\right) \boldsymbol{g}_{\xi}-\frac{1}{\mathrm{~J}}\left(\alpha_{\eta} \boldsymbol{g}_{\xi} \cdot \boldsymbol{g}_{\eta}+\alpha_{\xi} \boldsymbol{g}_{\xi} \cdot \boldsymbol{g}_{\xi}\right) \boldsymbol{g}_{\eta} \\
& =\frac{1}{\mathrm{~J}}\left[\left(\alpha_{\xi} \boldsymbol{g}_{\xi} \cdot \boldsymbol{g}_{\eta}+\alpha_{\eta} \boldsymbol{g}_{\eta} \cdot \boldsymbol{g}_{\eta}\right) \boldsymbol{g}_{\xi}-\left(\alpha_{\eta} \boldsymbol{g}_{\xi} \cdot \boldsymbol{g}_{\eta}+\alpha_{\xi} \boldsymbol{g}_{\xi} \cdot \boldsymbol{g}_{\xi}\right) \boldsymbol{g}_{\eta}\right],
\end{aligned}
$$

having recalled Eq. (2.3). Finally, the normal vectors are formulated in the following way:

$$
\begin{aligned}
& \mathrm{N}_{i j}^{e}=-\frac{\left(2 \boldsymbol{g}_{\eta}-\boldsymbol{g}_{\xi}\right)}{6} \times \boldsymbol{g}_{\zeta}=\frac{\left(\boldsymbol{g}_{\xi} \cdot \boldsymbol{g}_{\eta}-2 \boldsymbol{g}_{\eta} \cdot \boldsymbol{g}_{\eta}\right) \boldsymbol{g}_{\xi}+\left(2 \boldsymbol{g}_{\xi} \cdot \boldsymbol{g}_{\eta}-\boldsymbol{g}_{\xi} \cdot \boldsymbol{g}_{\xi}\right) \boldsymbol{g}_{\eta}}{6 \mathrm{~J}}, \\
& \mathrm{~N}_{j k}^{e}=-\frac{\left(-\boldsymbol{g}_{\eta}-\boldsymbol{g}_{\xi}\right)}{6} \times \boldsymbol{g}_{\zeta}=\frac{\left(\boldsymbol{g}_{\xi} \cdot \boldsymbol{g}_{\eta}+\boldsymbol{g}_{\eta} \cdot \boldsymbol{g}_{\eta}\right) \boldsymbol{g}_{\xi}-\left(\boldsymbol{g}_{\xi} \cdot \boldsymbol{g}_{\eta}+\boldsymbol{g}_{\xi} \cdot \boldsymbol{g}_{\xi}\right) \boldsymbol{g}_{\eta}}{6 \mathrm{~J}} \\
& \mathrm{~N}_{k i}^{e}=-\frac{\left(-\boldsymbol{g}_{\eta}+2 \boldsymbol{g}_{\xi}\right)}{6} \times \boldsymbol{g}_{\zeta}=\frac{\left(-2 \boldsymbol{g}_{\xi} \cdot \boldsymbol{g}_{\eta}+\boldsymbol{g}_{\eta} \cdot \boldsymbol{g}_{\eta}\right) \boldsymbol{g}_{\xi}+\left(-\boldsymbol{g}_{\xi} \cdot \boldsymbol{g}_{\eta}+2 \boldsymbol{g}_{\xi} \cdot \boldsymbol{g}_{\xi}\right) \boldsymbol{g}_{\eta}}{6 \mathrm{~J}} .
\end{aligned}
$$

\section{References}

[1] S. B. Savage, K. Hutter, The motion of a finite mass of granular material down a rough incline, J. Fluid Mech. 199 (1989) 177-215.

[2] M. Christen, J. Kowalski, and P. Bartelt, RAMMS: Numerical simulation of dense snow avalanches in three-dimensional terrain. Cold Regions Science and Technology, 63(1-2) (2010) 1-14. 
[3] R. P. Denlinger, R. M. Iverson, Granular avalanches across irregular three-dimensional terrain: 1. Theory and computation, Journal of Geophysical Research: Earth Surface 109 (F1) (2004) F01014.

[4] S. Li and C. J. Duffy, Fully coupled approach to modeling shallow water flow, sediment transport, and bed evolution in rivers, Water Resources Research, 47 (2011) W03508.

[5] Y.-S. Kuo, Y.-J. Tsai, Y.-S. Chen, C.-L. Shieh, K. Miyamoto, and T. Itoh, Movement of deepseated rainfall-induced landslide at Hsiaolin Village during Typhoon Morakot, Landslides, 10(2) (2013) 191-202.

[6] T. Takahashi, Debris flow: mechanics, prediction and countermeasures, CRC press, 2014.

[7] K. B. Khattri and S. P. Pudasaini, An extended quasi two-phase mass flow model, International Journal of Non-Linear Mechanics, 106 (2018), pp. 205-222.

[8] I. Luca, C.-Y. Kuo, K. Hutter, Y.-C. Tai, Modeling shallow over-saturated mixtures on arbitrary rigid topography, Journal of Mechanics, 28(3) (2012) 523-541.

[9] E. B. Pitman and L. Le, A two-fluid model for avalanche and debris flows, Philosophical Transactions of the Royal Society A: Mathematical, Physical and Engineering Sciences, 363 (2005), pp. 1573-1601.

[10] S. P. Pudasaini, A general two-phase debris flow model, Journal of Geophysical Research: Earth Surface, 117 (2012) F03010.

[11] S. P. Pudasaini and J.-T. Fischer, A mechanical model for phase-separation in debris flow, International Journal of Multiphase Flow, (2020), p. 103292.

[12] S. P. Pudasaini and M. Mergili, A multi-phase mass flow model. Journal of Geophysical Research: Earth Surface, 124 (2019) 2920-2942.

[13] M. Mergili, F. Jan-Thomas, J. Krenn, and S. P. Pudasaini, r.avaflow v1, an advanced opensource computational framework for the propagation and interaction of two-phase mass flows. Geoscientific Model Development 10 (2017) p. 553.

[14] M. Mergili, A. Emmer, A. Juřicová, A. Cochachin, J.-T. Fischer, C. Huggel, and S. P. Pudasaini. How well can we simulate complex hydro-geomorphic process chains? the 2012 multi-lake outburst flood in the Santa Cruz Valley (Cordillera Blanca, Perú). Earth Surface Processes and Landforms, 43(7) (2018) 1373-1389.

[15] M. Mergili, M. Jaboyedoff, J. Pullarello, and S. P. Pudasaini. Back calculation of the 2017 Piz Cengalo-Bondo landslide cascade with ravaflow: what we can do and what we can learn. Natural Hazards and Earth System Sciences, 20(2) (2020) 505-520.

[16] S. Savage, K. Hutter, The dynamics of avalanches of granular materials from initiation to runout. Part I: Analysis, Acta Mechanica 86 (1-4) (1991) 201-223.

[17] R. Greve, T. Koch, K. Hutter, Unconfined flow of granular avalanches along a partly curved surface. I. Theory, Proceedings of the Royal Society of London. Series A: Mathematical and Physical Sciences 445 (1924) (1994) 399-413.

[18] J. Gray, M. Wieland, K. Hutter, Gravity-driven free surface flow of granular avalanches over complex basal topography, Proc. R. Soc. Lond. A 455 (1999) 1841-1875.

[19] J. M. N. T. Gray, Y.-C. Tai, S. Noelle, Shock waves, dead zones and particle-free regions in rapid granular free-surface flows, Journal of Fluid Mechanics 491 (2003) 161-181.

[20] X. Meng, Y. Wang, Modelling and numerical simulation of two-phase debris flows, Acta Geotechnica 11 (5) (2016) 1027-1045.

[21] S. P. Pudasaini, K. Hutter, Rapid shear flows of dry granular masses down curved and twisted channels, Journal of Fluid Mechanics 495 (2003) 193-208.

[22] S. P. Pudasaini, Y. Wang, and K. Hutter, Modelling debris flows down general channels. Natural Hazards and Earth System Sciences, 5 (2005) 799-819. 
[23] S. P. Pudasaini, Y. Wang, L.-T. Sheng, S.-S. Hsiau, K. Hutter, and R. Katzenbach, Avalanching granular flows down curved and twisted channels: Theoretical and experimental results, Physics of Fluids 20 (2008), p. 073302.

[24] F. Bouchut, M. Westdickenberg, Gravity driven shallow water models for arbitrary topography, Comm. Math. Sci 2 (2004) 359-389.

[25] C.-Y. Kuo, Y.-C. Tai, F. Bouchut, A. Mangeney, M. Pelanti, R. Chen, K. Chang, Simulation of Tsaoling landslide, Taiwan, based on Saint Venant equations over general topography, Engineering Geology 104 (3-4) (2009) 181-189.

[26] I. Luca, Y.-C. Tai, and C.-Y. Kuo. Non-cartesian, topography-based avalanche equations and approximations of gravity driven flows of ideal and viscous fluids. Mathematical Models and Methods in Applied Sciences, 19 (01) (2009) 127-171.

[27] I. Luca, Y.-C. Tai, and C.-Y. Kuo, Modeling shallow gravity-driven solid-fluid mixtures over arbitrary topography, Communications in Mathematical Sciences 7 (2009) 1-36.

[28] W. Hui, P. Li, Z. Li, A unified coordinate system for solving the two-dimensional Euler equations, Journal of Computational Physics 153 (2) (1999) 596-637.

[29] W. H. Hui, The unified coordinate system in computational fluid dynamics, Communications in Computational Physics 2 (4) (2007) 577-610.

[30] Y.-C. Tai, C.-Y. Kuo, A new model of granular flows over general topography with erosion and deposition, Acta Mechanica 199 (2008) 71-96.

[31] Y.-C. Tai, Y.-C. Lin, A focused view of the behavior of granular flows down a confined inclined chute into horizontal run-out zone, Phys. Fluids 20 (2008) 123302.

[32] I. Luca, Y.-C. Tai, C.-Y. Kuo, Shallow geophysical mass flows down arbitrary topography, Springer, 2016.

[33] Y.-C. Tai, C.-Y. Kuo, Modelling shallow debris flows of the Coulomb-mixture type over temporally varying topography, Natural Hazards and Earth System Sciences 12 (2) (2012) 269280.

[34] Y.-C. Tai, C.-Y. Kuo, W.-H. Hui, An alternative depth-integrated formulation for granular avalanches over temporally varying topography with small curvature, Geophysical \& Astrophysical Fluid Dynamics 106 (6) (2012) 596-629.

[35] C.-Y. Kuo, Y.-C. Tai, C. Chen, K. Chang, A. Siau, J. Dong, R. Han, T. Shimamoto, C. Lee, The landslide stage of the Hsiaolin catastrophe: Simulation and validation, Journal of Geophysical Research: Earth Surface 116 (F04007) (2011) doi:10.1029 / 2010JF001921.

[36] Y.-C. Tai, J. Heß, Y. Wang, Modeling two-phase debris flows with grain-fluid separation over rugged topography: Application to the 2009 Hsiaolin event, Taiwan, Journal of Geophysical Research: Earth Surface 124 (2) (2019) 305-333.

[37] R. M. Iverson, Regulation of landslide motion by dilatancy and pore pressure feedback, Journal of Geophysical Research: Earth Surface 110 (2005) F02015.

[38] J. Heß, Y. Wang, K. Hutter, Thermodynamically consistent modeling of granular-fluid mixtures incorporating pore pressure evolution and hypoplastic behavior, Continuum Mechanics and Thermodynamics 29 (1) (2017) 311-343.

[39] J. Heß, Y.-C. Tai, Y. Wang, Debris flows with pore pressure and intergranular friction on rugged topography, Computers \& Fluids 190 (2019) 139-155.

[40] B. Braconnier, J.-J. Hu, Y.-Y. Niu, B. Nkonga, K.-M. Shyue, Numerical simulations of low Mach compressible two-phase flows: Preliminary assessment of some basic solution techniques., ESAIM: Proc. 28 (2009) 117-134.

[41] C.-Y. Kuo, B. Nkonga, M. Ricchiuto, Y.-C. Tai, B. Braconnier, Dry granular flows with erosion/deposition process., ESAIM: Proc. 28 (2009) 135-149. 
[42] M. Rauter, Ž. Tuković, A finite area scheme for shallow granular flows on three-dimensional surfaces., Computers \& Fluids 166 (2018) 184-199.

[43] C. Juez, D. Caviedes-Voullième, J. Murillo, P. García-Navarro, 2D dry granular free-surface transient flow over complex topography with obstacles. Part II: Numerical predictions of fluid structures and benchmarking, Computers \& Geosciences 73 (0) (2014) 142-163.

[44] I. Luca, K. Hutter, Y.-C. Tai, and C.-Y. Kuo, A hierarchy of avalanche models on arbitrary topography. Acta Mechanica 205 (2009) 121-149.

[45] M. Quecedo, M. Pastor, Finite element modelling of free surface flows on inclined and curved beds, Journal of Computational Physics 189 (1) (2003) 45-62.

[46] J.-T. Fischer, J. Kowalski, S. P. Pudasaini, Topographic curvature effects in applied avalanche modeling, Cold Regions Science and Technology 74 (2012) 21-30.

[47] E. Audusse, M. Bristeau, A well-balanced positivity preserving second-order scheme for shallow water flows on unstructured meshes, Journal of Computational Physics 206 (1) (2005) 311-333.

[48] G. Richard, S. Gavrilyuk, A new model of roll waves: comparison with Brock's experiments, Journal of Fluid Mechanics 698 (2012) 374-405.

[49] G. Richard, S. Gavrilyuk, The classical hydraulic jump in a model of shear shallow-water flows, Journal of Fluid Mechanics (2013) 492-521.

[50] A. Bhole, B. Nkonga, S. Gavrilyuk, K. Ivanova, Fluctuation splitting Riemann solver for a non-conservative modeling of shear shallow water flow, Journal of Computational Physics 392 (2019) 205-226.

[51] P. Chandrashekar, B. Nkonga, A. K. Meena, and A. Bhole, A path conservative finite volume method for a shear shallow water model, Journal of Computational Physics, (2020), p. 109457.

[52] O. Pouliquen, Y. Forterre, Friction law for dense granular flows: Application to the motion of mass down a rough inclined plane., J. Fluid Mech. 453 (2002) 133-151.

[53] G. Midi, On dense granular flows., Eur. Phys. J. E 14.

[54] E. Audusse, F. Bouchut, M. Bristeau, R. Klein, B. Perthame, A fast and stable well-balanced scheme with hydrostatic reconstruction for shallow water flows, SIAM J. Sci. Comput. 25 (2004) 2050-2065.

[55] G. Taubin, Estimating the tensor of curvature of a surface from a polyhedral approximation, Proceedings, Fifth International Conference on Computer Vision (1995) 902-907.

[56] J. J. Stoker, Water waves: The mathematical theory with applications, Vol. IV of Pure and applied mathematics, Interscience Publishers, Inc., New York, 1957.

[57] O. Delestre, C. Lucas, P.-A. Ksinant, F. Darboux, C. Laguerre, T.-N. Vo, F. James, S. Cordier, et al., Swashes: a compilation of shallow water analytic solutions for hydraulic and environmental studies, International Journal for Numerical Methods in Fluids 72 (3) (2013) 269-300.

[58] S. B. Savage, K. Hutter, The motion of a finite mass of granular material down a rough incline, Journal of fluid mechanics 199 (1989) 177-215.

[59] Y.-C. Tai, S. Noelle, J. Gray, K. Hutter, Shock-capturing and front-tracking methods for granular avalanches, Journal of Computational Physics 175 (1) (2002) 269-301.

[60] Y. Kuo, Y. Tsai, Y. Chen, C. Shieh, K. Miyamoto, T. Itoh, Movement of deep-seated rainfallinduced landslide at Shiaolin Village during typhoon Morakot, Landslides 10 (2) (2013) 191202.

[61] J. Dong, Y. Li, C. Kuo, R. Sung, M. Li, C. Lee, C. Chen, W. Lee, The formation and breach of a short-lived landslide dam at Hsiaolin village, Taiwan part I: post-event reconstruction of dam geometry, Engineering Geology 123 (1) (2011) 40-59. 
[62] A. E. Scheidegger, On the prediction of the reach and velocity of catastrophic landslides, Rock mechanics, 5 (1973), pp. 231-236.

[63] O. Castro-Orgaz, K. Hutter, J. V. Giraldez, W. H. Hager, Nonhydrostatic granular flow over 3-d terrain: New Boussinesq-type gravity waves?, Journal of Geophysical Research: Earth Surface 120 (1) (2015) 1-28.

[64] R. Kaitna, W. Dietrich, L. Hsu, Surface slopes, velocity profiles and fluid pressure in coarsegrained debris flows saturated with water and mud, Journal of Fluid Mechanics 741 (2014) 377-403.

[65] P.-Y. Lagrée, G. Saingier, S. Deboeuf, L. Staron, S. Popinet, Granular front for flow down a rough incline: about the value of the shape factor in depths averaged models, in: EPJ Web of Conferences, Vol. 140, EDP Sciences, 2017, p. 03046.

[66] R. Kaitna, M. C. Palucis, B. Yohannes, K. M. Hill, W. E. Dietrich, Effects of coarse grain size distribution and fine particle content on pore fluid pressure and shear behavior in experimental debris flows, Journal of Geophysical Research: Earth Surface 121 (2) (2016) 415-441.

[67] F. Bouchut, E. D. Fernández-Nieto, A. Mangeney, G. Narbona-Reina, A two-phase two-layer model for fluidized granular flows with dilatancy effects, Journal of Fluid Mechanics 801 (2016) 166-221.

[68] F. Bouchut, E. D. Fernández-Nieto, E. Koné, A. Mangeney, G. Narbona-Reina, A two-phase solid-fluid model for dense granular flows including dilatancy effects: comparison with submarine granular collapse experiments, in: EPJ Web of Conferences, Vol. 140, EDP Sciences, 2017, p. 09039. 